Further Evidence against Decay in Working Memory

Klaus Oberauer, University of Zurich

and

Stephan Lewandowsky, University of Bristol and University of Western Australia

Keywords: working memory, computational modeling, decay, interference

The research reported in this article was supported by a grant from Economic and Social Research Council UK (RES-062-23-1199) and a World University Network grant to the first author, and a Discovery Grant and a Discovery Outstanding Researcher Award from the Australian Research Council and a Wolfson Research Merit Award from the Royal Society to the second author.

Correspondence should be addressed to: Klaus Oberauer, University of Zurich, Department of Psychology - Cognitive Psychology; Binzmühlestrasse 14/22, 8050 Zürich, Switzerland, Email:

k.oberauer@psychologie.uzh.ch 


\begin{abstract}
Theories assuming that representations in working memory decay unless maintained by rehearsal must predict that in any condition where rehearsal does not fully counteract decay, memory declines with a longer retention interval. Two experiments served to test this prediction in a complex-span paradigm in which encoding of letters for later recall alternated with series of spatial distractor operations. The opportunity for rehearsal was varied through cognitive load, the proportion of available time per distractor operation that was actually needed for that operation. The time needed for each operation within a series was measured independently. Retention interval was varied by increasing the number of operations at constant cognitive load. Memory declined with higher cognitive load, but was unchanged (Experiment 1) or improved (Experiment 2) with more distractor operations. Decay theories can explain the effect of cognitive load by assuming that at high load rehearsal fails to fully compensate decay, but then must predict a negative effect of the number of operations at higher cognitive load, contrary to the data. Simulations with an interference-based computational model of working memory, SOB-CS, show that the model explains the experimental findings.
\end{abstract}




\section{Further Evidence against Decay in Working Memory}

When people try to remember a large amount of new information at the same time, they rapidly forget all but a small subset of it. The classic explanation for this phenomenon is that new information must initially be maintained in a short-term memory system - more recently referred to as working memory - that has a limited capacity (Atkinson \& Shiffrin, 1968). A popular explanation for this limited capacity is that representations in working memory rapidly decay over time, unless they are actively maintained by rehearsal. Because rehearsal takes time, and is applied to representations in working memory sequentially, the number of representations that can be protected from decay is limited by how many of them can be rehearsed before they fade away and become irrecoverable (Baddeley, 1986; Brown, 1958; Cowan \& AuBuchon, 2008; Kieras, Meyer, Mueller, \& Seymour, 1999; Page \& Norris, 1998; Reitman, 1974; Schweickert \& Boruff, 1986).

The popularity of the decay-rehearsal notion arises in part from its simplicity and intuitive appeal (Jonides et al., 2008). The hypothesis of trace decay in short-term or working memory has, however, met with serious criticism in several recent reviews (Jonides et al., 2008; Lewandowsky, Oberauer, \& Brown, 2009; Nairne, 2002). The most direct empirical challenge for decay theories comes from experiments in which performance on a test of working memory failed to decline over time, even though participants were kept busy with a rehearsal-preventing distractor task during the retention interval. For instance, a series of experiments showed that immediate recall of letter lists is unaffected by experimental manipulations of the intervals between individual letters at presentation or at recall, even when these intervals were filled with distracting tasks generally assumed to prevent rehearsal, such as articulatory suppression, choice response tasks, or a combination of both (Lewandowsky, Duncan, \& Brown, 2004; Lewandowsky, Geiger, Morrell, \& Oberauer, 2010; Lewandowsky, Geiger, \& Oberauer, 2008; Oberauer \& Lewandowsky, 2008, 2013).

Decay theories nevertheless do not fade away - rather, they adapt. One adaptive strategy involves the development of more potent mechanisms of rehearsal. One of the recent instantiations of decay theories, the time-based resource-sharing (TBRS) theory (Barrouillet, Portrat, \& Camos, 2011) 
comes equipped with a very powerful rehearsal mechanism, namely attention-based refreshing. As we will explain below, proponents of the TBRS theory invoke this mechanism to escape the empirical challenges confronted by more conventional decay theories. The first goal of the present article is to examine both logically and empirically whether the assumptions about refreshing incorporated in the TBRS theory actually protect decay theories against those empirical challenges. We will show that they do not.

Fortunately, decay theories of working memory are not without alternative. Theorists have proposed explanations of the capacity limit of working memory based on the assumption of a limited resource (Bays, Catalao, \& Husain, 2009; Just \& Carpenter, 1992; Keshvari, van den Berg, \& Ma, 2013), on the assumption of a limited number of discrete slots (Cowan, Rouder, Blume, \& Saults, 2012; Zhang \& Luck, 2008), and on the notion of interference between representations (Farrell \& Lewandowsky, 2002; Nairne, 1990; Oberauer \& Kliegl, 2001; Saito \& Miyake, 2004). Our approach is to investigate the most parsimonious explanation - arguably the one based on interference - and examine the extent to which it can explain forgetting in working memory. There is compelling evidence that interference plays a role in limiting how much we can remember over the short term: A large proportion of errors on working-memory tasks consist of confusions between items, and the probability of confusion increases systematically with the similarity between items in the memory set (Conrad \& Hull, 1964; Jalbert, Saint-Aubin, \& Tremblay, 2008; Lee \& Estes, 1977), with their proximity in a list (Henson, 1999; Murdock \& vom Saal, 1967), and with their spatial proximity in visual arrays (Rerko, Oberauer, \& Lin, in press). It is difficult to explain those findings without acknowledging that similar representations in memory interfere with each other. Interference by confusion clearly plays an important role in determining working-memory performance, and recent experiments are beginning to uncover evidence for other forms of interference as well, such as superposition of distributed representations and feature overwriting (Oberauer, Farrell, Jarrold, Pasiecznik, \& Greaves, 2012; Oberauer \& Lange, 2008). Therefore, we propose to explain the capacity limit of working memory by the processes of interference that can be empirically demonstrated to exist, and invoke additional explanatory constructs such as decay or resource limits only when necessary. Together with our colleagues we have developed a computational model of working 
memory that relies entirely on interference as the cause of forgetting, the SOB-CS model (Oberauer, Lewandowsky, Farrell, Jarrold, \& Greaves, 2012). Our second goal in this article is to demonstrate that this model accounts well for the data of our two new experiments, to be presented below, as well as published results that have been claimed to contradict the model.

In what follows we first review the TBRS theory and how well it can account for data from experiments in which the retention interval was varied while rehearsal was disrupted through distractor tasks. We then present two experiments that test a prediction following from the TBRS theory in a more controlled way than existing studies. Finally, we introduce SOB-CS as an alternative model, and show that a phenomenon that has been claimed to contradict SOB-CS and to support the TBRS theory in fact supports SOB-CS and contradicts the TBRS theory.

\section{The Time-Based Resource-Sharing (TBRS) Theory}

The core assumption of the TBRS theory is that representations in working memory decay unless they are refreshed by focusing attention on them. Attention is assumed to refresh representations one by one, unless it is engaged otherwise - for instance, by an attention-demanding distractor task. The TBRS theory has been applied primarily to the complex-span paradigm, in which encoding of items of a memory list alternates with brief episodes of distractor activity. For instance, encoding of consonants might alternate with series of parity judgments on digits (Barrouillet, Bernardin, Portrat, Vergauwe, \& Camos, 2007). Each parity judgment is assumed to demand attention, but in between individual digits, attention is assumed to instantly switch to refreshing memory traces. Every brief time gap in between two distractor operations is thought to be used for refreshing. As a consequence, this version of decay theory - in contrast to earlier instantiations of the decay hypothesis - does not predict that memory necessarily declines over a retention interval filled with a distractor task. If the gaps between distractor operations are long enough, and refreshing is fast enough, decay can be fully counteracted by refreshing.

The relation between decay, refreshing, and distraction of attention is characterized by an equation that defines the cognitive load (CL) imposed by the distracting activity: 
$\mathrm{CL}=a N / T$,

where $a$ is the duration for which each distractor operation (e.g., each parity judgment) captures attention, $N$ is the number of successive distractor operations in each distractor episode of a complex-span task, and $T$ is the time available for all distractor operations in that episode. Because CL is the ratio of two time variables, it can be manipulated independently of the absolute duration of a distractor-filled retention interval. For instance, CL can be manipulated by the rate at which attentiondemanding operations are required, even if the total time available for the distractor task remains invariant. Conversely, the length of distractor-filled intervals can be increased without changing CL by increasing the total number of distractor steps while holding their rate constant. This can be formally expressed by rewriting the total time $T$ as the product of the number of distractors $N$ and the time available for each distractor, $t$, the latter reflecting the rate of distractor operations:

$$
\mathrm{CL}=a N / t N
$$

Because $N$ appears in the numerator and the denominator, it can be increased without changing CL. This insight led Barrouillet and colleagues to make two predictions from the TBRS theory: First, memory declines with increasing CL. Second, as long as CL is held constant, the number of distractorprocessing steps, and with it the duration of the retention interval, has no effect on memory. Both predictions have been confirmed several times (Barrouillet, Bernardin, \& Camos, 2004; Barrouillet et al., 2007; Barrouillet, Portrat, \& Camos, 2011; Barrouillet, Portrat, Vergauwe, Diependaele, \& Camos, 2011), lending strong prima-facie support to the TBRS theory.

The second prediction - that the length of a distractor-filled retention interval has no effect on memory as long as CL is held constant - is an elegant way of escaping the main empirical challenge for decay theories. It has therefore been invoked by Barrouillet and his colleagues to fend off criticism of the decay assumption (Barrouillet, Portrat, Vergauwe, et al., 2011). They argue that previous demonstrations of constant memory performance across variations of distractor-filled delay (e.g., Lewandowsky et al., 2004; Oberauer \& Lewandowsky, 2008) are fully compatible with the TBRS theory. We next show that this claim is wrong. 


\section{The Effect of Number of Distractors on Memory: Re-Evaluating the TBRS Theory}

Does the TBRS theory warrant the prediction that the number of distractor operations has no effect on memory in complex-span tests when CL is constant? A theory makes a prediction if and only if the prediction follows logically from its assumptions. Here we make explicit the premises that are required to deduce the above prediction. The argument leading to this prediction is based on the following logic: (1) The core assumptions of the TBRS theory imply that complex-span performance depends on CL. If the further assumption is made that (2) only CL systematically affects memory performance, then it follows that if CL is held constant, performance is unaffected by the number of operations. The second step in the argument, that performance depends only on CL in a systematic fashion, has never been made explicit, but it is a necessary assumption to provide a logical bridge from the premises of the TBRS theory to the prediction by Barrouillet and colleagues about the number of distractor operations. To highlight the necessity of this bridging assumption, imagine that performance is a function not only of CL, but also of a factor X exerting a systematic influence in addition to CL: If that were the case, then keeping CL constant could not be taken to imply that performance remained unchanged unless $\mathrm{X}$ was also controlled. The bridging assumption - that there is no such factor $\mathrm{X}$, or $\mathrm{Y}$, or Z-is therefore an essential, if tacit, aspect of the reasoning underlying the presumed predictions from the TBRS theory. We now show that this bridging assumption is incompatible with the premises of the TBRS theory.

\section{Formal Analysis and Simulations}

Our analysis begins with a decomposition of the processes presumed by the TBRS theory when a distractor operation is required and their predicted effects on memory strength (see Figure 1 for illustration): At any level of CL, each distractor operation $i$ captures attention for duration $a_{i}$. This attentional-capture time is followed by a period of free time $f t_{i}$ that can be used for refreshing of memoranda. The total time $T$ in the CL equation is the sum of $a_{i}$ and $f t_{i}$ across all distractors $i[1 \ldots N]$ within a distractor episode, that is, within the retention interval between presentation of two successive memoranda. During each interval $a_{i}$ there is a certain loss of memory strength $\Delta \mathrm{d}_{\mathrm{i}}$ by decay without refreshing to mitigate it. During each free time period $f t_{i}$ there is a gain in memory strength $\Delta \mathrm{r}_{\mathrm{i}}$ 
generated by refreshing. The net loss of memory strength from processing a distractor $i$ therefore is $\Delta \mathrm{d}_{\mathrm{i}}$ $-\Delta \mathrm{r}_{\mathrm{i} .}$ Let $\Delta \mathrm{D}$ be the mean loss of memory strength through decay across all distractor operations in a distractor episode, and $\Delta \mathrm{R}$ the corresponding mean gain of memory strength through refreshing. Then the net loss after $N$ distractors equals $N(\Delta \mathrm{D}-\Delta \mathrm{R})$. If, at a given level of CL, $\Delta \mathrm{D}$ exceeds $\Delta \mathrm{R}$, then the net loss of memory strength necessarily increases with $N$, which inevitably leads to the prediction of more forgetting with increasing number of distractor operations at a constant level of CL. Thus, memory must be predicted to depend not only on CL but also on a second variable, $N$.

The only exception to this conclusion arises when $\Delta \mathrm{R}$ is equal to $\Delta \mathrm{D}$, such that refreshing fully compensates the loss incurred by decay-because it is only when $\Delta \mathrm{D}-\Delta \mathrm{R}=0$ that $N$ no longer matters. However, setting $\Delta \mathrm{R}$ equal to $\Delta \mathrm{D}$ has a further implication: when refreshing exactly offsets any decay during processing, then distractor processing cannot impair memory at all compared to a no-distractor control: In the TBRS theory, the only effect distractor processing is assumed to have on memory is to prevent refreshing. Therefore, when $\Delta R=\Delta D$, performance in complex-span tasks must be predicted to be equal to performance in simple-span tasks in which no distractor processing is requested (see panels A-C of Figure 1). Empirically, this prediction is rarely borne out: Complex-span performance is virtually always worse than simple-span performance. Logically, the assumption that $\Delta \mathrm{D}-\Delta \mathrm{R}=0$ can only hold for one particular (very low) level of $\mathrm{CL}, \mathrm{CL}_{0}$, for which complex-span performance is predicted to equal simple-span performance. For any level of CL higher than $\mathrm{CL}_{0}, \Delta \mathrm{D}$ $\Delta \mathrm{R}$ must be larger than zero, implying that memory performance must be predicted to decline with increasing $N$ (see panels D and E of Figure 1).

We buttressed this formal analysis by simulation, using a computational implementation of the TBRS theory, TBRS* (Oberauer \& Lewandowsky, 2011). The TBRS* model incorporates the three premises of the TBRS theory together with fairly generic assumptions about the representations underlying serial recall. Confirming a core prediction of the TBRS theory, our simulations produced an approximately linear effect of CL on memory performance. However, in confirmation of our preceding analysis and in contradiction to the presumed informal predictions of the TBRS theory, TBRS* was found always to produce more forgetting with a greater number of distractors, except for 
conditions of very low CL, when complex-span performance approached simple-span performance. Figure 2 shows the relevant simulation results (for details of this simulation, see Oberauer \& Lewandowsky, 2011).

Our formal analysis and simulations with TBRS* thus converge on the conclusion that the TBRS theory necessarily predicts a negative effect of the number of distractors on complex-span performance at all but the lowest CLs.

\section{A first Experimental Test}

Barrouillet, Portrat, Vergauwe, et al. (2011) present an experiment disconfirming that prediction of the TBRS theory. In their study, participants remembered lists of letters, interspersed with processing episodes that required a series of two-alternative choice tasks (i.e., deciding by key press whether a square was displayed above or below the screen midline). The study manipulated the number of successive choice operations (four or eight) and the available time per operation $(t=780$, 980, or $1180 \mathrm{~ms}$ ). Barrouillet et al.'s results are reproduced in the left panel of Figure 3. Letter recall decreased as available time per operation decreased, but recall was not significantly affected by the number of successive choices at all three paces; numerically, recall was even better with eight than with four operations.

Within the TBRS framework, the fact that recall declined with pace implies that at least at the faster two paces (i.e., greater CL levels), refreshing was incomplete, and therefore $\Delta R<\Delta D$. Although $\Delta R$ and $\Delta D$ are difficult to estimate for a single level of $C L$, when an experiment contains multiple $C L$ levels, and performance is found to decline with increasing load, then the equality $\Delta R=\Delta \mathrm{D}$ that is required for the number of operations to have no effect can only hold for the lowest CL, if at all. At the larger CL levels, $\Delta \mathrm{R}<\Delta \mathrm{D}$ must be true, because otherwise the CL manipulation would have had no effect. It follows that the TBRS theory must predict a negative effect of the number of operations for any but the lowest level of CL, and the absence of that effect therefore challenges the model.

To support this analysis, we applied the TBRS* model to the experiment by Barrouillet, Portrat, Vergauwe, et al. (2011) using the published set of standard parameter values (Oberauer \& 
Lewandowsky, 2011) that works best for most applications, including the simulation of the CL effect (Figure 2). To apply TBRS*, we need an estimate of the duration for which each choice operation captures attention, thereby preventing refreshing. Based on previous studies, Barrouillet and colleagues estimated this time to be $390 \mathrm{~ms}$ for the task used in their 2011 study. This estimate was well matched by the response times of their experiment, and we therefore used that time in the simulation. All other specifications (i.e., list length, timing of events) were taken directly from the experiment. Figure 3 (center panel) shows the results of the simulation. In confirmation of our conceptual analysis, at each level of CL, TBRS* predicts more forgetting with eight than with four operations, contrary to the data of Barrouillet, Portrat, Vergauwe, et al. (2011). This result challenges the claim of Barrouillet and colleagues that their theory was compatible with the data they reported.

There is, however, a potential loophole for the TBRS theory. Although in the experiment of Barrouillet, Portrat, Vergauwe, et al. (2011) all distractor operations in a distractor episode were nominally equivalent - they were all randomly selected instances of the same two-choice decision task - it is not necessarily the case that they all capture attention for the same amount of time. It is conceivable that the first operation (i.e., the first decision), or the first few operations, in a distractor episode demand more attention than subsequent operations. If this were the case, then CL would decline across successive operations within a distractor episode. As a consequence, the average CL in shorter distractor episodes would be higher than in longer ones. This speculation receives some evidence from response times of distractor operations at different sequential positions in a distractor episode: Response times were substantially longer for the first operation than for all subsequent operations in an episode (Oberauer \& Lewandowsky, 2013). This observation mirrors the commonly observed time cost of switching between tasks (Monsell, 2003): Participants need to switch from encoding a memory item to doing the distractor task, and that switch appears to cost time. If task switching is attention demanding, then the first operation captures attention longer than any subsequent operation. This implies a larger CL during the first operation than on subsequent operations as long as the available time is held constant across all operations, as was the case in the study of Barrouillet and colleagues. One could therefore argue that the condition with fewer operations was at a disadvantage because it imposed a larger average CL. This disadvantage would have 
counteracted the decline of memory with a larger number of distractor operations that the TBRS theory must predict. We therefore carried out two experiments testing the effect of number of operations on complex-span performance with a tighter control of CL.

\section{Experiment 1: Four vs. Eight Distractors}

We tested performance in a complex span task, independently varying CL and the number of distractor operations per distractor episode (four or eight). The complex-span task was preceded by a test of the time demands of distractor operations as a function of their sequential position within a distractor episode. In the memory task we individually adjusted for each participant the time available for each distractor to the distractor's time demand, separately for each sequential position in the episode. In this way we held CL constant across all within-episode positions, and hence, constant between short and long distractor episodes.

\section{Method}

Participants. Twenty students from University of Zurich took part in a three-session experiment in exchange for course credit or monetary compensation (45 CHF, approximately 50 USD). Data from 14 trials of one participant were lost due to technical failure.

Materials and Procedure. Participants were tested on a complex span task. The memory task consisted of serial recall of lists of seven letters. Lists were created by drawing randomly without replacement from the 20 consonants of the German alphabet. Each letter was presented centrally on the screen for $1 \mathrm{~s}$. The distractor task was a spatial decision task: The stimulus for each decision was a horizontal bar presented above or below a pair of horizontally aligned squares. The participant's task was to decide whether the bar fits in the gap between the squares. A substantial effect of CL with this task has been observed for both verbal and spatial memoranda (Vergauwe, Barrouillet, \& Camos, 2010).

In each trial of the complex-span task, presentation of letters (for $1 \mathrm{~s} \mathrm{each)} \mathrm{alternated} \mathrm{with}$ distractor episodes of four or eight spatial-decision operations. Trials started and ended with a distractor episode. Participants were instructed to read each letter aloud and to remember all letters in 
order. They were informed that they had limited time for each distractor response. The available time per distractor operation was determined according to the CL level of the memory trial, based on the person's time demand for the distractor task (see below). After the final distractor episode participants were asked to recall the seven letters in forward order by typing them in response to seven sequential prompts on the screen. They were not allowed to skip a letter.

In the first session we measured response times (RTs) for the spatial-decision task without memory load. Participants were exposed to sequences of events mirroring those in the complex-span task: Presentation of letters (for $1 \mathrm{~s}$ ) alternated with distractor episodes of eight spatial-decision operations. Each sequence of events consisted of seven letters embedded in eight distractor episodes, corresponding to one complex-span trial. Participants were asked to read each letter aloud, but not to remember the letters. They were instructed to make the spatial decisions as quickly and accurately as possible, pressing the left arrow key for "does fit" and the right arrow key for "does not fit" judgments. Distractor stimuli stayed on the screen until participants responded. After each response the next distractor, or the next letter, was presented immediately. There were 32 replications of the event sequence, each containing eight distractor episodes with eight distractors each. We measured distractor RTs and determined for each participant the median RT for each sequential position in a distractor episode; each median was based on 256 responses.

From the median RTs in the first session we calculated the time available per distractor operation in the subsequent two sessions of complex-span testing, separately for each participant and each within-episode position, as follows: The maximum display duration of the distractor stimulus was set to 1.5 times the median RT in the high CL condition, and to 3 times the median RT in the low $\mathrm{CL}$ condition. To this time we added 0.2 times the maximum display duration during which the screen stayed blank, but responses were still recorded, before the next distractor stimulus, or the next letter, was presented. Thus, the available time $t_{i}$ for a distractor operation in within-episode position $i$ was 1.2 x $1.5 \times$ median $\left(\mathrm{RT}_{\mathrm{i}}\right)$ for high $\mathrm{CL}$, and $1.2 \times 3.0 \times$ median $\left(\mathrm{RT}_{\mathrm{i}}\right)$ for low CL. Each distractor stimulus stayed on the screen until the person responded or until the maximum display duration was reached. During the remainder of the available time $t_{i}$ the screen stayed blank. In each of the two sessions with 
complex-span testing, participants completed 32 test trials ( 8 per condition), preceded by 4 practice trials.

\section{Results}

Distractor Task. We first analyzed the data from the distractor task during complex-span trials to assess how successfully we controlled CL across conditions. Table 1 provides an overview of the proportion of time-out errors (i.e., distractors to which no response was given in the available time) and of incorrect responses. We included RTs from correct and incorrect responses in the RT analyses because distractor operations leading to incorrect responses most likely captured attention as much as those resulting in correct responses. RTs to distractors with time-out errors were set to the available time for the respective distractor operation because we assumed that in those cases participants continued processing the distractor until the end of the available time. ${ }^{1}$

The top panel of Figure 4 plots the mean distractor RTs during complex-span trials (sessions 2 and 3) by CL level, number of distractor operations, and position within the distractor episode. The averaged median RTs obtained in the calibration test (without memory task, session 1) are plotted in red. These data showed four features. First, RTs in the first position were substantially elevated, replicating our earlier observation (Oberauer \& Lewandowsky, 2013). The effect was present not only during the complex-span trials but also during the calibration test, demonstrating that it is independent of a concurrent memory load. This observation rules out the possibility that participants postpone responding to the first distractor to squeeze in some rehearsal activity because in the calibration test there was nothing to rehearse. The most likely explanation of the increased RTs in the first position is that they reflect a task-switch cost.

Second, RTs during complex span were shorter when CL was higher. This is most likely a reflection of the higher time pressure when CL was high. Third, RTs in the short and the long distractor episodes in the complex-span test did not differ when compared in the same within-episode position. Fourth, during the calibration test RTs slightly increased during an episode across positions 2 to 8 , whereas during the complex-span task, they slightly decreased. These different trends could be due to the time pressure in the complex-span trials or due to the memory load, but we cannot think of 
any compelling reason why either of these variables should lead to an acceleration of RTs within a distractor episode.

We calculated CL for each participant in each condition at each within-episode position by dividing the mean distractor RTs by the available time. The bottom panel of Figure 4 presents these CL estimates. CL clearly differs between the two levels of our CL manipulation, as intended. By calibrating available time to the time demand estimated in the first session, we compensated for the switch cost in RTs, and consequently, CL differed very little between positions. Because of the diverging trends in the calibration test and the complex-span test, CL nevertheless slightly declined across positions. As a consequence of this decline, average CL was slightly higher for the short than the long distractor episodes. As we will show below, this difference was much too small to bias the effect of number of distractors on memory.

Memory. Proportion of letters recalled in their correct positions is plotted as a function of CL and number of distractor operations in Figure 5; the serial-position curves for the four conditions can be found in the Appendix. The placement of the data points on the x-axis of Figure 5 reflects the CL estimates calculated above. In line with previous findings, CL had a large effect on memory performance. Contrary to the predictions from the TBRS theory, memory did not decline with increasing number of distractor operations - if anything, memory was slightly better with eight than with four operations at the higher CL level.

We assessed the strength of evidence for the effects of CL and number of distractor operations on memory through a Bayesian ANOVA (Rouder, Morey, Speckman, \& Province, 2012) using the BayesFactor 0.9.0 package (Morey \& Rouder, 2012) for R (R-Development-Core-Team, 2012). The package calculates the Bayes factor for comparison of two ANOVA models. The Bayes factor is the Bayesian likelihood ratio of the two models under comparison. It specifies to what extent the ratio of the prior probabilities for the two models should be updated in light of the data to obtain the ratio of posterior probabilities. If the two models have equal prior probability, the Bayes factor expresses the ratio of their posterior probabilities, that is, it indicates how much more probable one model is compared to the other in light of the data. Bayes factors $<3$ are usually regarded as evidence "barely 
worth mentioning"; BF between 3 and 10 as "substantial evidence", BF between 10 and 100 as "strong evidence", and BF > 100 as "decisive" (Kass \& Raftery, 1995).

In a first step, we compared two models, each with a main effect of one of the two independent variables, to the null model without any effect. The Bayes factor in favor of the model with the main effect of CL was 47,583, providing decisive evidence for an effect of CL. The Bayes factor in favor of the model with the main effect of number of operations was 0.54 , which if anything speaks in favor of the null model. The Bayes factor for the null model is the reciprocal of the Bayes factor for the alternative model, $1 / 0.54=1.85$. In a second step, we evaluated the evidence for the interaction of CL and number of operations by comparing a model with the main effect of CL and the interaction to a model with only the main effect of $\mathrm{CL}$, omitting the interaction. Both models omitted the main effect of number of operations, which received no support in the initial analysis. The Bayes factor in favor of the interaction was 1.34. This value is ambiguous because it reflects roughly equal evidence for both models. Fortunately, the interaction is of no theoretical significance because it does not discriminate between competing theoretical claims.

The Bayesian ANOVA tests the null hypothesis against a two-sided alternative hypothesis. Thus, the Bayes factor in favor of the null effect of number of operations, 1.85, quantifies the strength of evidence of the null model over an alternative model allowing any effect of number of operations, be it positive or negative. If anything, the effect reflected in our data was positive. A Bayesian estimate of the probable direction and size of the effect is available from the posterior probability density of the effect of number of distractors (Kruschke, 2011), shown in Figure $6 .{ }^{2}$ More than $95 \%$ of the posterior probability mass lies over positive values; positive values imply that increasing the number of distractor operations has a positive effect on memory.

Wagenmakers and Morey (2013) explain how to calculate the Bayes factor for comparing the null hypothesis to a one-sided alternative hypothesis. We used their method to obtain the Bayes factor for a model with a negative effect of number of operations against the null model; it was 0.054. Its reciprocal, 18.6, quantifies the evidence in favor of the null model against the model with a negative 
effect. This value reflects strong evidence for the null model stating that the number of operations has no effect on memory performance.

\section{Discussion}

The response times for distractor operations confirmed the suspicion that the duration of attentional capture by nominally equivalent distractors is not homogeneous within a distractor episode. The first distractor requires more time because of the need to switch from memory encoding to distractor processing. This fact implies that previous experiments testing the effect of number of distractor operations on memory did not fully control CL. In our experiment we compensated the switch cost with a longer time window for the first operation, thereby reducing the difference in CL between short and long distractor episodes to a negligible minimum. To assess the effect of the remaining difference, one can visually shift the data points for 4 operations and 8 operations in Figure 5 along the slope of the CL effect (i.e., the lines connecting the points) until they are aligned on the x-axis. It is obvious that this shift does not change the results in any substantive way.

The memory data confirm that increasing the number of distractor operations while holding CL constant has no detrimental effect on memory. If anything, the effect is beneficial. Our finding is in full agreement with the finding of Barrouillet, Portrat, Vergauwe, et al. (2011), who also observed better memory performance with 8 than with 4 operations (see the left panel of Figure 3), although that effect was not significant in their data. For comparison with the present results we calculated the Bayes factor comparing a model with an effect of number of operations to a null-effect model from the F value reported by Barrouillet and colleagues, using Jeff Rouder's online Bayes-Factor Calculator (http://pcl.missouri.edu/bf-one-sample). The Bayes factor was 2.8 in favor of an effect, which is weak evidence for a beneficial effect of more operations. Unlike the study of Barrouillet and colleagues, we controlled CL more precisely between the two levels of the number-of-operations variable.

Nevertheless, we obtained largely equivalent results. The findings from both experiments contradict the prediction that logically follows from the premises of the TBRS theory; namely, that at greater CLs performance should decline with more operations. 
One limitation of our first experiment is that comparing 4 and 8 operations is not the strongest possible test of the prediction of the TBRS theory. We chose these two conditions to facilitate the comparison with the experiment of Barrouillet, Portrat, Vergauwe, et al. (2011), but the simulations in Figure 2 show that the effect of number of operations predicted by TBRS* is relatively small for 4 versus 8 operations. It is much larger for the contrast of 1 versus 4 operations. The reason why the effect of number of operations becomes smaller at larger numbers of operations is that the opposing effects of decay and refreshing on memory performance eventually reach a steady state across several operations, and the closer the system gets to that steady state, the less effect is predicted for a further increase of number of operations (Oberauer \& Lewandowsky, 2011). Therefore, in Experiment 2 we compared 1 versus 4 operations at the same level of CL to obtain a stronger test of the prediction of the TBRS theory that more operations lead to worse memory.

\section{Experiment 2}

\section{Method}

Participants. Twenty-four students of University of Zurich took part in a single session; they received either course credit or $15 \mathrm{CHF}$ as reimbursement.

Materials and Procedure. Participants were tested on a complex span task exactly as in Experiment 1, with the only difference that the number of distractor operations (i.e., spatial decisions) preceding and following each letter was 1 or 4 . Participants completed 48 complex-span trials (12 per condition), preceded by 8 practice trials. Different from Experiment 1 we did not measure each participant's space-judgment RTs in a pre-test without memory load. Rather, we used the median RTs of the pre-test in Experiment 1, averaged over participants, to calibrate the presentation durations for spatial decisions in the complex-span task. The available time $t_{i}$ for a distractor operation in withinepisode position $i$ was $1.2 \times 1.5 \times$ mean $\left(\operatorname{median}\left(\mathrm{RT}_{\mathrm{i}}\right)\right)$ for high $\mathrm{CL}$, and $1.2 \times 3.0 \times$ mean $\left(\operatorname{median}\left(\mathrm{RT}_{\mathrm{i}}\right)\right)$ for low CL. 


\section{Results}

Distractor Task. The proportion of time-outs and errors in the distractor task are summarized in Table 1. The top panel of Figure 7 shows response times for the spatial fit judgments, and the bottom panel shows the CL values calculated from these RTs and the available time for each distractor operation. The RTs were slightly longer than the means of the median RTs from the pre-test in Experiment 1 (the red line in Figure 7) probably because participants in Experiment 1 had more practice with the spatial decision task. Nevertheless, the resulting CL values are fairly constant across within-episode positions, so that CL differed only minimally between trials with 1 and with 4 operations. This small difference went in the opposite direction from that in Experiment 1, with slightly lower CL for 1 than for 4 operations.

Memory. Recall accuracy is plotted in Figure 8; serial-position curves are given in the Appendix. Memory performance declined with increasing CL $(B F=92.5)$, and was better with 4 operations than with 1 operation $(\mathrm{BF}=15.4)$. Evidence with regard to the interaction was ambiguous; the Bayes Factor in favor of the interaction was 1.5. Figure 9 shows the posterior density of the effect of number of operations. The mass of this density is clearly concentrated over positive values, implying that the posterior probability of a zero or negative effect is negligible.

\section{Discussion}

For the contrast of one versus four operations, the TBRS theory predicts a substantial decrement of memory with more operations. The data reflected the opposite effect: Memory was better with four operations than with one. This result is incompatible with the TBRS theory. To further buttress this conclusion, we ran additional simulations with TBRS*.

\section{Simulation with TBRS*}

As we show in the introduction, the prediction of an impairment of memory with more operations follows logically from the premises of the TBRS theory. The only way in which the theory can predict a null effect of number of operations is by having refreshing fully compensate decay. In that case there cannot be an effect of CL. To further demonstrate this relationship between the two 
predicted effects we ran simulations with TBRS*, varying the strength of re-encoding of items through refreshing (i.e., encoding rate parameter $R$ ); all other parameters were set to their standard values, except for decay rate $=0.4$. We simulated the design of our experiments with 1,4 , and 8 operations, at two levels of CL, set to the CL values obtained in Experiment 1. Figure 10 shows the predictions from TBRS* for Experiments 1 and 2 using the standard parameter value of $R=6$. The model predicts a strong negative effect of CL, and an equally strong negative effect of number of operations. The results of varying encoding rate $R$ are summarized in Figure 11. The left panel shows the effect of number of operations as a function of $R$. The predicted effect is negative throughout, but it approaches zero at the highest values of $R$, when refreshing is very powerful. The right panel shows the corresponding effects of the CL manipulation. These effects are mostly negative, except at the lowest and the highest values of $R$. At the highest encoding rates, refreshing fully compensates decay, and performance is close to ceiling regardless of CL and of number of operations. At very low encoding rates, refreshing is too weak to have a tangible impact, and the effect of CL disappears. Critically, there is no value of $R$ at which the effect of number of operations at high CL approaches zero while the effect of CL is still negative. This simulation result illustrates our argument in the introduction: Whenever there is a negative effect of CL, the net effect of decay and refreshing must be negative, implying a negative effect of number of operations (see also Figure 1). It is impossible for a model in which memory performance depends on the balance of decay and refreshing (or any other kind of rehearsal) to produce a negative CL effect without a negative effect of number of operations - but this is precisely the pattern we obtained in both our experiments, and that Barrouillet and colleagues (2011) obtained before.

To summarize, we showed in two experiments that increasing the number of attentiondemanding distractors while holding CL constant has no detrimental effect on memory. Our finding converges with numerous previous reports that show unimpaired memory as a distractor-filled retention interval is increased, albeit not always with tight controls of CL (Lewandowsky et al., 2004; Lewandowsky et al., 2010; Lewandowsky et al., 2008; Oberauer \& Lewandowsky, 2008, 2013; Vallar \& Baddeley, 1982). These findings jointly provide strong evidence against decay in working memory. Therefore, we believe it is time to turn our attention to an alternative to decay theories. 


\section{An Interference-Based Theory: SOB-CS}

The SOB-CS model (Oberauer, Lewandowsky, et al., 2012) is an extension of the C-SOB model of serial recall (Farrell, 2006; Lewandowsky \& Farrell, 2008) to the complex-span paradigm. The model is based on four assumptions: (1) There is no time-based decay in working memory; forgetting arises entirely from interference between distributed representations, (2) distractor processing impairs memory because it engages representations that are involuntarily encoded into working memory, thereby adding to interference, (3) the strength of encoding of any information into working memory depends on its novelty, with contextually novel information being encoded more strongly than familiar information (i.e., encoding is "novelty-gated"), and (4) interfering representations can be gradually removed from working memory (i.e., they are "unbound" from their context; see Kessler \& Meiran, 2008) when there is time to do so before other information needs to be processed.

The fourth assumption enables SOB-CS to explain the approximately linear decline of memory performance with increasing CL as follows: CL can be increased by either decreasing the available time $t$, or by increasing the duration of attentional capture by the distractor operations, $a$. Reducing $t$ reduces the time available for removing distractor representations, resulting in more interference; increasing $a$ leads to stronger encoding of distractors, again resulting in more interference. In SOB-CS, the latter effect is predicted to be relatively small—compared to the larger effect of free time — because distractor encoding saturates more quickly than removal; this prediction has been confirmed experimentally (Oberauer, Lewandowsky, et al., 2012).

We have previously used C-SOB to derive predictions for the effect of the number of distractors on performance in complex span (Lewandowsky et al., 2010; Lewandowsky et al., 2008). The assumption of novelty-gated encoding plays an important role for these predictions: If the distractors in an episode involve processing different materials (e.g., reading several different words), memory declines as the number of distractor operations increases, because every distractor operation involves representations that are to some degree novel, and therefore are encoded with a nonnegligible strength, adding to interference with the memoranda. In contrast, if the distractor operations 
all involve the same (or very similar) material (e.g., repeatedly reading the same word), their number has no effect on memory, because the representations involved in successive distractor operations have negligible novelty, so that hardly any interference is added after the first distractor operation in each episode. We confirmed this prediction in several experiments (Lewandowsky et al., 2010; Lewandowsky et al., 2008; see also Macken \& Jones, 1995) in which we asked participants to remember lists of letters. Following either encoding or recall of each letter, participants had to read aloud either a single word, three different words, or repeat the same word multiple times. Articulating the same word repeatedly did not lead to any further impairment of memory over and above reading a single word, but reading three different words resulted in additional forgetting.

These successful predictions of C-SOB notwithstanding, Barrouillet, Portrat, Vergauwe, et al. (2011) claimed that their finding of no effect of number of distractor operations contradicted the CSOB model. They argued that any model which—such as C-SOB and also SOB-CS—assumes novelty-gated encoding of distractors and some restorative process during free time in between distractors, must predict better memory with 8 than with 4 distractors. Their rationale was that only the first distractor in an episode (but not the remaining ones) is encoded to a non-negligible degree, so that the amount of interference is the same irrespective of the number of distractors. In contrast, the total time available for memory restoration increases with the number of distractors, doubling between 4 and 8 distractors. Given the assumption that interference remains constant, more restoration time should lead to better memory with 8 than with 4 distractor operations.

This argument is an over-simplification that neglects how the effects of novelty-gated encoding and removal play off against each other under different conditions of CL and of interdistractor similarity. In SOB-CS, novelty of each stimulus is assessed relative to an expectation generated by the memory content previously associated to the present context. In a serial-recall task the context is the serial position of each list item, which is not advanced by distractors; thus all distractors following an item are associated to the position of that item (direct evidence for this assumption has been reported by Oberauer, Farrell, et al., 2012). As a consequence, the first distractor following each item is novel to the extent that it differs from the preceding item - in the present case, 
the representations involved in spatial judgments are without doubt sufficiently different from the preceding letter to result in strong encoding. After completing the first distractor operation, the representations generated by it are gradually removed from memory (i.e., unbound from their context) during the free time remaining until onset of the next distractor. What happens with regard to further distractor operations depends on the $\mathrm{CL}$ and on the similarity between successive distractors:

In the extreme case where $\mathrm{CL}$ is so high that distractors cannot be removed at all, the effect of subsequent distractors depends only on how similar they are to the first distractor: If they are all identical, novelty-gated encoding prevents encoding of all but the first distractor, so that the total amount of interference does not change as more distractors are added. The more dissimilar subsequent distractors are, the more novel each of them is, leading to stronger encoding of each distractor, and interference increases with the number of distractors. Hence, the effect of number of distractors on memory is predicted to be negative. These predictions have been derived through simulation and confirmed empirically before (Lewandowsky et al., 2010; Lewandowsky et al., 2008; Oberauer, Lewandowsky, et al., 2012). The predictions and results are sufficiently well established to require little further argument; they establish that when distractors processed at high CL are dissimilar from each other, they result in cumulative interference, generating a negative effect of a larger number of distractors, whereas there is no negative effect of number of distractors that are all identical.

The novel question of interest here is what happens when CL is reduced, leaving some free time for distractor removal. During the free time following the first distractor, its representation is gradually removed. With lower CL (i.e., more free time) removal becomes increasingly complete. When subsequent distractors are identical to the first, they are again novel in the present context (i.e., relative to the current position marker) to the extent that the previous distractor has been removed. The more the preceding distractor has been removed, the more the next distractor is encoded, even if it is identical to the preceding one. When distractors are dissimilar from each other, the extent of removal matters less, because each distractor is encoded, and adds interference, due to its novelty independent of the removal of the preceding distractor. 
The cycle of encoding a distractor, removing it, and encoding the next distractor continues for as many times as distractors follow a memory item. It is inadvisable to anticipate the net effect of increasing the number of distractors on memory under these conditions without performing simulations. We therefore carried out simulations with SOB-CS for the present experimental paradigm. Application of the SOB-CS model to this paradigm requires two decisions about the representations of the distractors. First, the representational overlap between verbal memoranda and the representations involved in a spatial distractor task is arguably low, but it cannot be assumed to be zero. ${ }^{3}$ Doing the spatial-decision task involves more than generating a spatial representation of the stimuli - it also involves holding a representation of the stimulus-response mappings in working memory, and making a decision about the response, as well as a largely unknown set of representations involved in executive control (for a detailed treatment of the overlap between memoranda and distractors in complex span, including a simulation varying the degree of overlap, see Oberauer, Lewandowsky, et al., 2012). We therefore set the overlap (i.e., proportion of feature units shared) between representations of memory items and of distractors to $50 \%$. The second decision concerns the similarity between successive distractors. The individual spatial decisions are highly similar to each other but not identical. The most important difference between them pertains to the decision: For half the stimuli the bar fits into the gap and for the other half, it does not. Obviously these two kinds of stimuli require different representations. In our simulation we therefore used two representations for the distractors, one for "does fit" and one for "does not fit" stimuli, choosing one for each simulated distractor operation at random with equal probability. ${ }^{4}$ Although we find it plausible that these two distractor representations are highly similar to each other, we initially varied inter-distractor similarity (i.e., the vector cosine of the two representations) over a broad range to explore its effect.

We simulated the paradigm of our experiments with 1,4 , or 8 spatial decisions as distractors. The duration of each distractor was set to the mean RT observed in Experiment 1 for each withinepisode position; the available time was set to the mean of the total times assigned to each position in both experiments. We varied CL over three levels: The two levels realized in Experiment 1, plus an extreme level with $\mathrm{CL}$ of 1 , leaving no free time for removal. The latter load condition enables a direct comparison of the present simulations with those in our previous studies, in which CL was very high 
and we assumed no distractor removal (Lewandowsky et al., 2010). Finally, we varied similarity between distractors over the entire possible range, from completely dissimilar (cosine $=0)$ to identical $(\operatorname{cosine}=1)$ in steps of 0.1 .

The results are shown in Figure 12. The right panel plots the effect of 1 versus 4 operations as a function of inter-distractor similarity for three levels of CL: The low and high CL conditions in Experiment 1 , and $\mathrm{CL}=1$. The condition with $\mathrm{CL}=1$ replicated the previous simulations with $\mathrm{C}-\mathrm{SOB}$ and SOB-CS: With highly similar or identical distractors, number of operations had virtually no effect; the more dissimilar the distractors were from each other, the more negative the effect of number of operations, reflecting worse memory with 4 than with 1 operation. At the CL levels of the present experiment, which allow for some degree of distractor removal, the effect of number of operations was positive with high inter-distractor similarity, and this positive effect was larger for the higher of these two CL levels. Thus, for this particular combination of inter-distractor similarity and CL, the simulations confirm the intuitive prediction made by Barrouillet, Portrat, Vergauwe, et al. (2011). This prediction matches the findings of the present experiments. With decreasing similarity between distractors, the effect of number of operations turned negative again, exactly as observed in previous studies that manipulated distractor similarity (Lewandowsky et al., 2010; Lewandowsky et al., 2008).

The right panel of Figure 12 plots the effects of CL (low versus high level of Experiment 1) as a function of inter-distractor similarity separately for 1,4 , and 8 operations. The effect of CL is always negative, but its size is modulated by inter-distractor similarity when there are 4 or 8 distractors in each processing episode. In SOB-CS, higher CL implies that interference can be remedied to a lesser extent by distractor removal. More dissimilar distractors produce more interference, so that there is more to be gained from removing distractors, resulting in a larger benefit of low compared to high CL. With a single distractor operation, inter-distractor similarity has little effect because distractor similarity affects only the encoding strength of distractors following the first one in a processing episode (Lewandowsky et al., 2008).

As explained above, we can assume the similarity between distractor representations for "does fit" and "does not fit" decisions to be high but not perfect; we therefore chose 0.8 as a realistic value. 
Figure 13 shows the simulation results with that value. They correspond well with the experimental data: Performance declined with increasing CL, and was better with 4 or 8 distractors compared to 1 , especially at high CL. For completeness, we also ran a simulation of the experiment of Barrouillet, Portrat, Vergauwe, et al. (2011), using the same inter-distractor similarity value. The results are shown in the right panel of Figure 3. Again, memory is predicted to decline with CL, but unlike the TBRS* model, SOB-CS predicts no difference between 4 and 8 operations. This prediction matches the findings reported by Barrouillet and colleagues. To conclude, the results of the experiment of Barrouillet and colleagues imply the opposite of what those authors claim: The null effect of number of operations contradicts the TBRS theory but is in full agreement with the SOB-CS model.

\section{General Discussion}

Decay theories of working memory must predict that memory performance declines over an increasing retention interval, unless decay is assumed to be fully counteracted by some form of rehearsal. If it is assumed that, in a given experimental condition, rehearsal fully counteracts decay, then decay theories cannot predict any memory loss through decay in that condition. We applied this general logic to the TBRS theory (Barrouillet, Portrat, \& Camos, 2011). The TBRS theory receives its main empirical support from the well-established finding that memory accuracy in complex-span paradigms declines with increasing cognitive load (CL). According to the TBRS theory, the effect of $\mathrm{CL}$ arises because with increasing CL, refreshing - an attention-based form of rehearsal - is increasingly less able to compensate decay. If this explanation holds, it follows that whenever an experiment shows an effect of CL, at the higher level of CL the effect of decay is not fully compensated by rehearsal. As a consequence, the theory must predict a negative effect of increasing the retention interval (i.e., increasing the number of distractors) at that level of CL. Our two experiments contradict that prediction, as does a previous experiment by Barrouillet, Portrat, Vergauwe, et al. (2011). These results imply that at least one core assumption of the TBRS theory is wrong: Either there is no decay in working memory, or the CL effect has nothing to do with the relative strength of decay and rehearsal. 
Although we developed our argument with a focus on the TBRS theory, it applies generally to all theories assuming decay and some compensatory process of rehearsal or restoration of memory representations. For any given experimental condition, the amount of memory loss through decay occurring in an arbitrarily selected retention interval is either larger than the compensatory gain from rehearsal or restoration, or it is not. If the loss from decay is larger, then it must be the case that increasing the retention interval under the same conditions leads to more forgetting. If the loss from decay is not larger than the compensatory gain, then decay has no effect on performance, and therefore cannot be invoked to explain any empirical phenomenon, such as the effect of CL. This argument holds regardless of which process of rehearsal or restoration is assumed to counteract decay. For instance, researchers could assume that in addition to attention-based refreshing, people engage in articulatory rehearsal to maintain verbal memoranda (Camos, Lagner, \& Barrouillet, 2009), and because our distractor task was non-verbal, there was nothing to prevent them from rehearsing the letters. If this is the case, we must again ask: Is the combined effect of articulatory rehearsal and refreshing at the higher level of CL sufficient to fully compensate the effect of decay? If it is not, the theory must still predict a negative effect of increasing the retention interval from 1 to 4 to 8 operations. If it is, then the theory has no way to explain why performance is worse at higher CL.

We conclude that our findings challenge a broad class of theories, namely all theories that (1) assume decay and some form of rehearsal or restoration, and (2) explain the CL effect through the balance of decay and rehearsal or restoration. Among existing decay theories of working memory, there is none that explains the CL effect in another way, and therefore, our findings are incompatible with extant decay theories.

Fortunately, there are promising alternatives to decay theories of working memory. We showed through simulation that one interference theory, the SOB-CS model, explains both the effect of CL and the simultaneous absence of a negative effect of the number of distractor operations. Assuming high inter-distractor similarity - as is plausible for the present experiments - the model predicts even a small beneficial effect of increasing the number of operations. This effect was numerically observed in both our experiments and the study by Barrouillet, Portrat, Vergauwe, et al. 
(2011), and received strong statistical evidence in our Experiment 2. SOB-CS also accounts for a range of other findings from simple and complex span tasks (Lewandowsky \& Farrell, 2008; Lewandowsky et al., 2010; Oberauer, Lewandowsky, et al., 2012). No other contemporary model can explain the joint effects of cognitive load and number of distractor operations. Therefore, the present results strongly support the SOB-CS model. 
Appendix

Serial-position curves for accuracy of recall in complex span can be found in Figure A1 for Experiment 1, and in Figure A2 for Experiment 2 


\section{Footnotes}

1) We also ran the analyses of distractor RTs of Experiments 1 and 2 excluding error and time-out trials. This led to slightly shorter mean RTs and therefore to smaller CL estimates (by no more than 0.03 ) because of the exclusion of the relatively long time-out RTs, but the pattern of results did not change.

2) The posterior density of any parameter of a Bayesian model can be obtained from the Markov-Chain Monte-Carlo (MCMC) chains that contain the simulated posteriors. We computed the MCMC chains with the ImBF function of the BayesFactor package, running the full ANOVA model with both main effects and interaction. The chains for the three effects were obtained with the posterior function. The plot was produced with the function HDIofMCMC provided by Kruschke (2011).

3) Overlap does not imply similarity; overlap refers to shared feature dimensions whereas similarity refers to shared feature values. For instance, a carrot and a lime overlap on the feature dimensions color, size, shape, and acidity, among many others, but they are similar only on one of these dimensions (i.e., size). In contrast, a carrot and the ringtone of a telephone have less overlap because ringtones have no color, size, shape, or acidity. In SOB-CS, carrots and limes are represented as different patterns of activity over the same units, whereas carrots and ringtones are represented in largely non-overlapping sets of units. Understanding the difference between overlap and similarity in distributed memory networks is essential to understand how very dissimilar representations can interfere with each other. In SOB-CS there are two forms of interference, one arising from confusion, depending on similarity, and the other arising from superposition, depending on the proportion of overlap. When memoranda and distractors come from different categories, such as letters and spatial judgments, their similarity is minimal, so they will never be confused; yet they can interfere by superposition to the extent that their representations overlap (Oberauer, Farrell, et al., 2012; Oberauer, Lewandowsky, et al., 2012).

4) In their footnote 1, Barrouillet, Portrat, Vergauwe et al. (2011) argued that the two stimuli in their distractor task (judging stimuli as being above or below the midline) must be regarded as 
identical, on the grounds that we stated (Lewandowsky \& Oberauer, 2009) that difficult decisions (where the stimuli were closer to the midline) created no more interference than easy decisions (where they were separated further). There is no logical connection between the two statements: In both the easy and the difficult conditions, the two kinds of stimuli differ, and they must be represented as different (i.e., as "above" vs. "below") because otherwise the task could not be done. 
Atkinson, R. C., \& Shiffrin, R. M. (1968). Human memory: A proposed system and its control processes. In K. W. Spence \& J. T. Spence (Eds.), The psychology of learning and motivation: Advances in research and theory (Vol. 2, pp. 90-195). New York: Academic Press.

Baddeley, A. D. (1986). Working memory. Oxford: Clarendon Press.

Barrouillet, P., Bernardin, S., \& Camos, V. (2004). Time constraints and resource sharing in adults' working memory spans. Journal of Experimental Psychology: General, 133, 83-100.

Barrouillet, P., Bernardin, S., Portrat, S., Vergauwe, E., \& Camos, V. (2007). Time and cognitive load in working memory. Journal of Experimental Psychology: Learning, Memory \& Cognition, 33, 570-585.

Barrouillet, P., Portrat, S., \& Camos, V. (2011). On the law relating processing to storage in working memory. Psychological Review, 118, 175-192.

Barrouillet, P., Portrat, S., Vergauwe, E., Diependaele, K., \& Camos, V. (2011). Further evidence for temporal decay in working memory: Reply to Lewandowsky and Oberauer (2009). Journal of Experimental Psychology: Learning, Memory, and Cognition, 37, 1302-1317.

Bays, P. M., Catalao, R. F. G., \& Husain, M. (2009). The precision of visual working memory is set by allocation of a shared resource. Journal of Vision, 9, 1-11.

Brown, J. (1958). Some tests of the decay theory of immediate memory. Quarterly Journal of Experimental Psychology, 10, 12-21.

Camos, V., Lagner, P., \& Barrouillet, P. (2009). Two maintenance mechanisms of verbal information in working memory. Journal of Memory and Language, 61, 457-469.

Conrad, R., \& Hull, A. J. (1964). Information, acoustic confusion and memory span. British Journal of Pychology, 55, 429-432.

Cowan, N., \& AuBuchon, A. M. (2008). Short-term memory loss over time without retroactive stimulus interference. Psychonomic Bulletin \& Review, 15, 230-235.

Cowan, N., Rouder, J. N., Blume, C. L., \& Saults, J. S. (2012). Models of verbal working memory capacity: What does it take to make them work? Psychological Review, 119, 480-499 doi: 10.1037/a0027791

Farrell, S. (2006). Mixed-list phonological similarity effects in delayed serial recall. Journal of Memory and Language, 55, 587-600.

Farrell, S., \& Lewandowsky, S. (2002). An endogenous distributed model of ordering in serial recall. Psychonomic Bulletin \& Review, 9, 59-79.

Henson, R. N. A. (1999). Positional information in short-term memory: Relative or absolute? Memory \& Cognition, 27, 915-927.

Jalbert, A., Saint-Aubin, J., \& Tremblay, S. (2008). Visual similarity in short-term recall for where and when. Quarterly Journal of Experimental Psychology, 61, 353-360.

Jonides, J., Lewis, R. L., Nee, D. E., Lustig, C. A., Berman, M. G., \& Moore, K. S. (2008). The mind and brain of short-term memory. Annual Review of Psychology, 59, 193-224.

Just, M. A., \& Carpenter, P. A. (1992). A capacity theory of comprehension: Individual differences in working memory. Psychological Review, 99, 122-149.

Kass, R. E., \& Raftery, A. E. (1995). Bayes Factors. Journal of the American Statistical Association, 90, 773-795.

Keshvari, S., van den Berg, R., \& Ma, W. J. (2013). No evidence for an item limit in change detection. PLoS Computational Biology, 9. Retrieved from doi:10.1371/journal.pcbi.1002927

Kessler, Y., \& Meiran, N. (2008). Two dissociable updating processes in working memory. Journal of Experimental Psychology: Learning, Memory, and Cognition, 34, 1339-1348.

Kieras, D. E., Meyer, D. E., Mueller, S., \& Seymour, T. (1999). Insights into working memory from the perspective of the EPIC architecture for modeling skilled perceptual-motor and cognitive human performance. In A. Miyake \& P. Shah (Eds.), Models of working memory. Mechanisms of active maintenance and executive control (pp. 183-223). Cambridge: Cambridge University Press. 
Kruschke, J. K. (2011). Doing Bayesian data analysis: A tutorial with R and BUGS. New York: Academic Press.

Lee, C. L., \& Estes, W. K. (1977). Order and position in primary memory for letter strings. Journal of Verbal Learning \& Verbal Behavior, 16, 395-418.

Lewandowsky, S., Duncan, M., \& Brown, G. D. A. (2004). Time does not cause forgetting in short-term serial recall. Psychonomic Bulletin \& Review, 11, 771-790.

Lewandowsky, S., \& Farrell, S. (2008). Short-term memory: new data and a model. In B. H. Ross (Ed.), The Psychology of Learning and Motivation (Vol. 49, pp. 1-48). London, UK: Elsevier.

Lewandowsky, S., Geiger, S. M., Morrell, D. B., \& Oberauer, K. (2010). Turning simple span into complex span: time for decay or interference from distractors? Journal of Experimental Psychology: Learning Memory and Cognition, 36(4), 958-978.

Lewandowsky, S., Geiger, S. M., \& Oberauer, K. (2008). Interference-based forgetting in verbal shortterm memory. Journal of Memory and Language, 59(2), 200-222.

Lewandowsky, S., \& Oberauer, K. (2009). No evidence for temporal decay in working memory. Journal of Experimental Psychology-Learning Memory and Cognition, 35(6), 1545-1551.

Lewandowsky, S., Oberauer, K., \& Brown, G. D. A. (2009). No temporal decay in verbal short-term memory. Trends in Cognitive Sciences, 13(3), 120-126.

Macken, W. J., \& Jones, D. M. (1995). Functional characteristics of the inner voice and the inner ear: Single or double agency. Journal of Experimental Psychology: Learning, Memory, and Cognition, 21, 436-448.

Monsell, S. (2003). Task switching. Trends in Cognitive Sciences, 7, 134-140.

Morey, R. D., \& Rouder, J. N. (2012). BayesFactor (Version 0.8.8). Retrieved from http://cran.at.rproject.org/web/packages/BayesFactor/index.html

Murdock, B. B., \& vom Saal, W. (1967). Transpositions in short-term memory. Journal of Experimental Psychology, 74, 137-143.

Nairne, J. S. (1990). A feature model of immediate memory. Memory \& Cognition, 18, 251-269.

Nairne, J. S. (2002). Remembering over the short-term: The case against the standard model. Annual Review of Psychology, 53, 53-81.

Oberauer, K., Farrell, S., Jarrold, C., Pasiecznik, K., \& Greaves, M. (2012). Interference between maintenance and processing in working memory: The effect of item-distractor similarity in complex span Journal of Experimental Psychology: Learning, Memory, and Cognition, 38, 665685. doi: 10.1037/a0026337

Oberauer, K., \& Kliegl, R. (2001). Beyond resources: Formal models of complexity effects and age differences in working memory. European Journal of Cognitive Psychology, 13(1-2), 187-215.

Oberauer, K., \& Lange, E. B. (2008). Interference in verbal working memory: Distinguishing similaritybased confusion, feature overwriting, and feature migration. Journal of Memory and Language, 58(3), 730-745.

Oberauer, K., \& Lewandowsky, S. (2008). Forgetting in immediate serial recall: Decay, temporal distinctiveness, or interference? Psychological Review, 115(3), 544-576.

Oberauer, K., \& Lewandowsky, S. (2011). Modeling working memory: a computational implementation of the Time-Based Resource-Sharing theory. Psychonomic Bulletin \& Review, $18(1), 10-45$.

Oberauer, K., \& Lewandowsky, S. (2013). Evidence against decay in verbal working memory. Journal of Experimental Psychology: General, 142, 380-411.

Oberauer, K., Lewandowsky, S., Farrell, S., Jarrold, C., \& Greaves, M. (2012). Modeling working memory: An interference model of complex span. Psychonomic Bulletin \& Review, 19, 779819.

Page, M. P. A., \& Norris, D. (1998). The primacy model: A new model of immediate serial recall. Psychological Review, 105, 761-781.

R-Development-Core-Team. (2012). R: A language and environment for statistical computing (Version 2.15.2). Vienna, Austria: R Foundation for Statistical Computing. Retrieved from URL: http://www.R-project.org 
Reitman, J. S. (1974). Without surrepetitious rehearsal, information in short-term memory decays. Journal of Verbal Learning and Verbal Behavior, 13, 365-377.

Rerko, L., Oberauer, K., \& Lin, H.-Y. (in press). Spatially imprecise representations in working memory. Quarterly Journal of Experimental Psychology.

Rouder, J. N., Morey, R. D., Speckman, P. L., \& Province, J. M. (2012). Default Bayes factors for ANOVA designs. Journal of Mathematical Psychology, 56, 356-374.

Saito, S., \& Miyake, A. (2004). On the nature of forgetting and the processing-storage relationship in reading span performance. Journal of Memory and Language, 50, 425-443.

Schweickert, R., \& Boruff, B. (1986). Short-term memory capacity: Magic number or magic spell? Journal of Experimental Psychology: Learning, Memory, and Cognition, 12, 419-425.

Vallar, G., \& Baddeley, A. D. (1982). Short-term forgetting and the articulatory loop. Quarterly Journal of Experimental Psychology, 34A, 53-60.

Vergauwe, E., Barrouillet, P., \& Camos, V. (2010). Do mental processes share a domain-general resource? Psychological Science, 21, 384-390.

Wagenmakers, E.-J., \& Morey, R. D. (2013). Simple relation between one-sided and two-sided Bayesian point-null hypothesis tests. Unpublished Manuscript, University of Amsterdam.

Zhang, W., \& Luck, S. J. (2008). Discrete fixed-resolution representations in visual working memory. Nature, 453, 233-236. 
Table 1: Percentage of Time-Out Errors and Errors in Distractor Operations

\begin{tabular}{|l|l|l|l|l|}
\hline & \multicolumn{2}{|l|}{ Experiment 1 } & \multicolumn{2}{l|}{ Experiment 2 } \\
\hline & 4 Operations & 8 Operations & 1 Operation & 4 Operations \\
\hline Time-Outs, low CL & 0.6 & 0.7 & 0.4 & 0.3 \\
\hline Time-Outs, high CL & 4.0 & 2.8 & 4.2 & 3.8 \\
\hline Errors, low CL & 5.2 & 5.5 & 3.3 & 4.1 \\
\hline Errors, high CL & 7.4 & 7.3 & 8.3 & 9.4 \\
\hline
\end{tabular}


Figure Captions

Figure 1. The interplay of decay and refreshing in the time-based resource-sharing (TBRS) theory. A: Simple span (no distractor processing): Memory strength of item M1 remains undiminished at least until encoding of the following item M2. B and C: Complex span at very low CL: Decay during distractor processing (triangles marked P) is fully offset by refreshing during free time (triangles marked R). Memory strength of M1 remains undiminished until encoding of M2, as in simple span. As a consequence, predicted memory performance is equal for zero distractors (A), two distractors (B), and four distractors (C). D and E: Complex span at higher CL, such that refreshing only partially offsets decay. Memory strength declines with every processing-refreshing cycle, and therefore declines more with four operations (E) than with two operations (D).

Figure 2: TBRS* simulation of memory span as a function of CL and number of operations (from Oberauer \& Lewandowsky, 2011). CL was manipulated by crossing three levels of attentional-capture duration $(0.3,0.5$, and $0.7 \mathrm{~s})$ with five levels of free time $(0,0.1,0.6,1.2$, and $2.0 \mathrm{~s})$. The condition with 0 operations reflects simple span, for which CL is not defined; therefore the 15 data points reflect 15 replications of the same simulation.

Figure 3. Data from the experiment of Barrouillet, Portrat, Vergauwe, et al. (2011) are displayed in the left panel. Memory declined with increasing pace; the apparent advantage for eight over four operations was not statistically significant. The middle panel shows predictions from TBRS* generated with the published parameter values (Oberauer \& Lewandowsky, 2011), except that the decay rate was decreased from 0.5 to 0.4 to bring overall performance in correspondence with the data. The right panel shows predictions from SOB-CS generated with the published parameter values (Oberauer, Lewandowsky, et al., 2012); similarity between distractors was 0.8 .

Figure 4. Top: Distractor RTs from Experiment 1. Bottom: Estimates of cognitive load for Experiment 1.

Figure 5. Memory performance in Experiment 1. Error bars are Bayesian 95\% highest-density intervals (Kruschke, 2011) 
Figure 6. Posterior probability density of the effect of number of distractor operations on memory in Experiment 1 . The black bar covers the $95 \%$ highest-density interval (HDI). The HDI indicates the narrowest range of parameter values that covers $95 \%$ of the posterior probability mass (Kruschke, 2011). In other words, in light of the data the true value of the effect has a probability of .95 to lie within the HDI.

Figure 7. Top: Distractor RTs from Experiment 2. Bottom: Estimates of cognitive load for Experiment 1.

Figure 8. Memory performance in Experiment 2. Error bars are Bayesian 95\% highest-density intervals (Kruschke, 2011).

Figure 9. Posterior probability density of the effect of number of distractor operations on memory in Experiment 2. The black bar covers the 95\% highest-density interval (HDI).

Figure 10. Predictions from simulation with TBRS* for Experiments 1 and 2, using standard parameter values, except for decay rate $=0.4$.

Figure 11. Predictions from simulation with TBRS*. Left: Effect of number of operations (1 vs. 4) on memory (proportion correct) as a function of encoding rate. Right: Effect of cognitive load (high vs. low as in Experiment 1) on memory as a function of encoding rate.

Figure 12. Predictions from simulation with SOB-CS. Effect of number of operations (1 vs. 4$)$ on memory (proportion correct) as a function of inter-distractor similarity. Right: Effect of cognitive load (high vs. low as in Experiment 1) on memory as a function of inter-distractor similarity.

Figure 13. Predictions from simulation with SOB-CS for Experiments 1 and 2, using standard parameter values. Representational overlap between letters and distractor representations $=50 \%$; interdistractor similarity $=0.8$. 
Figure 1

A
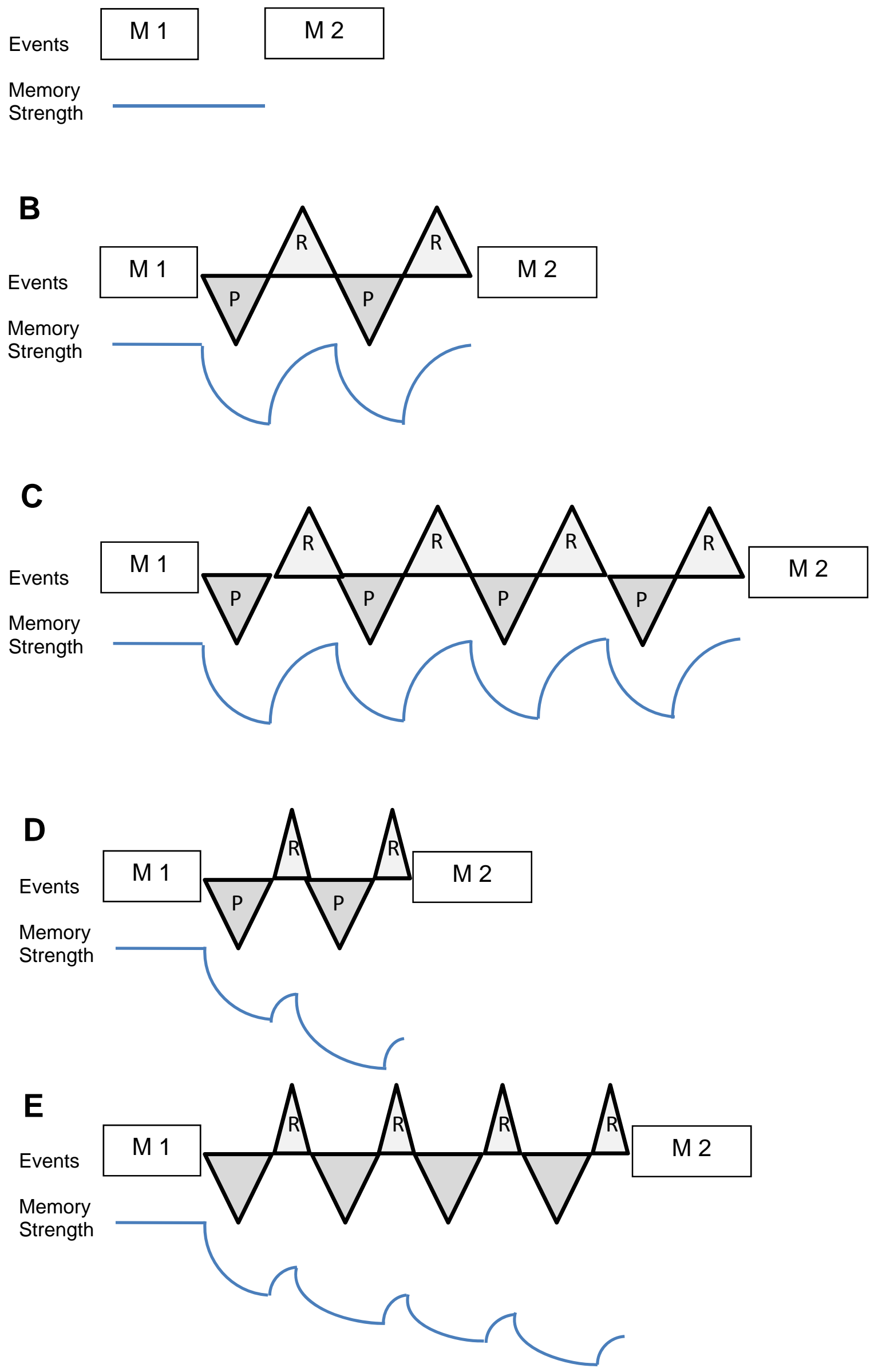
Figure 2

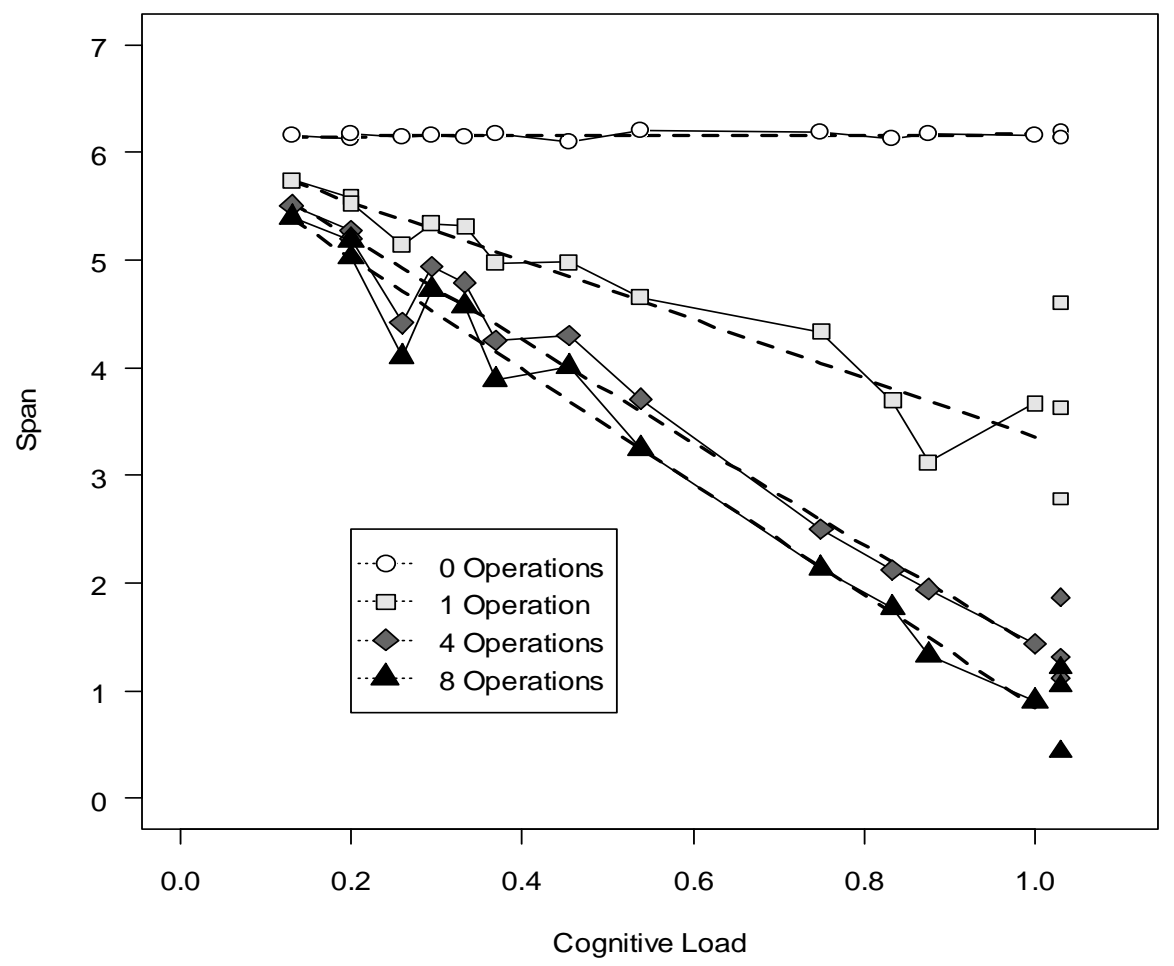


Figure 3
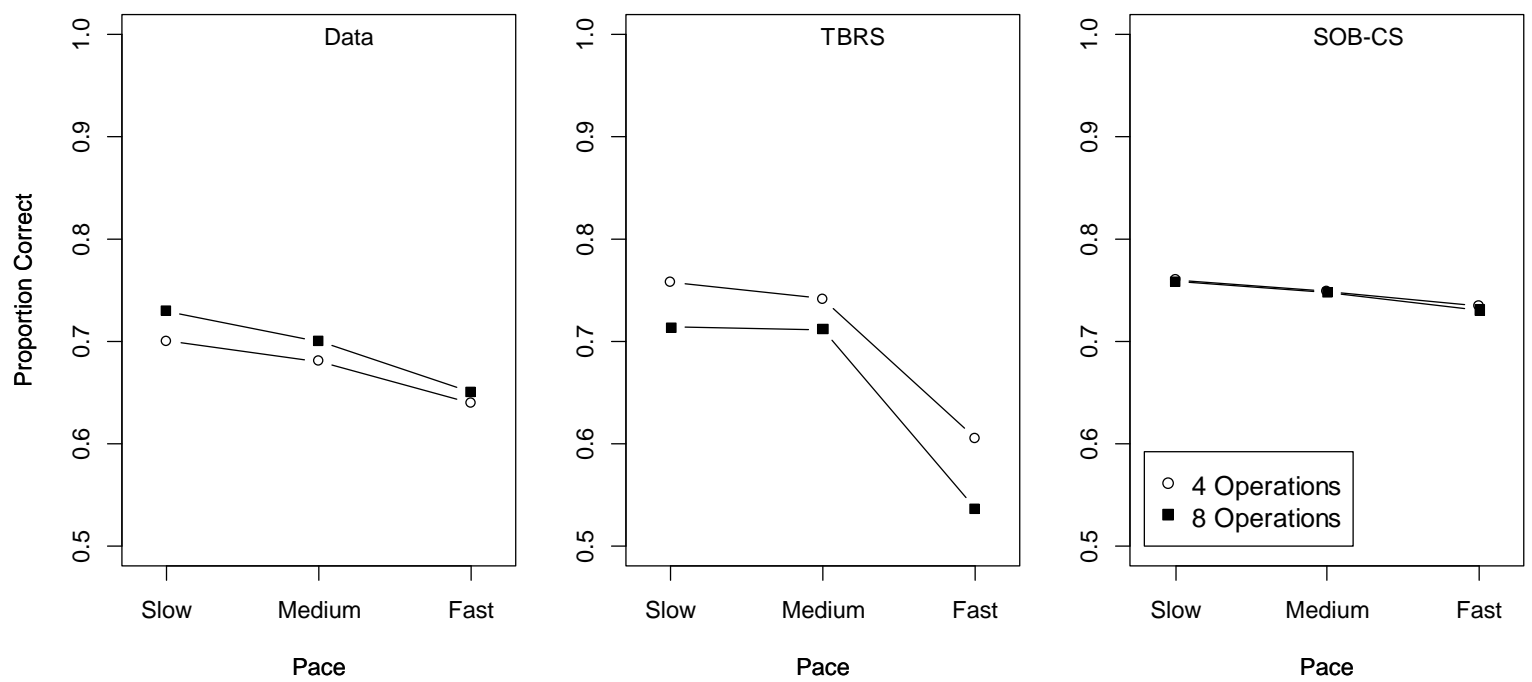
Figure 4
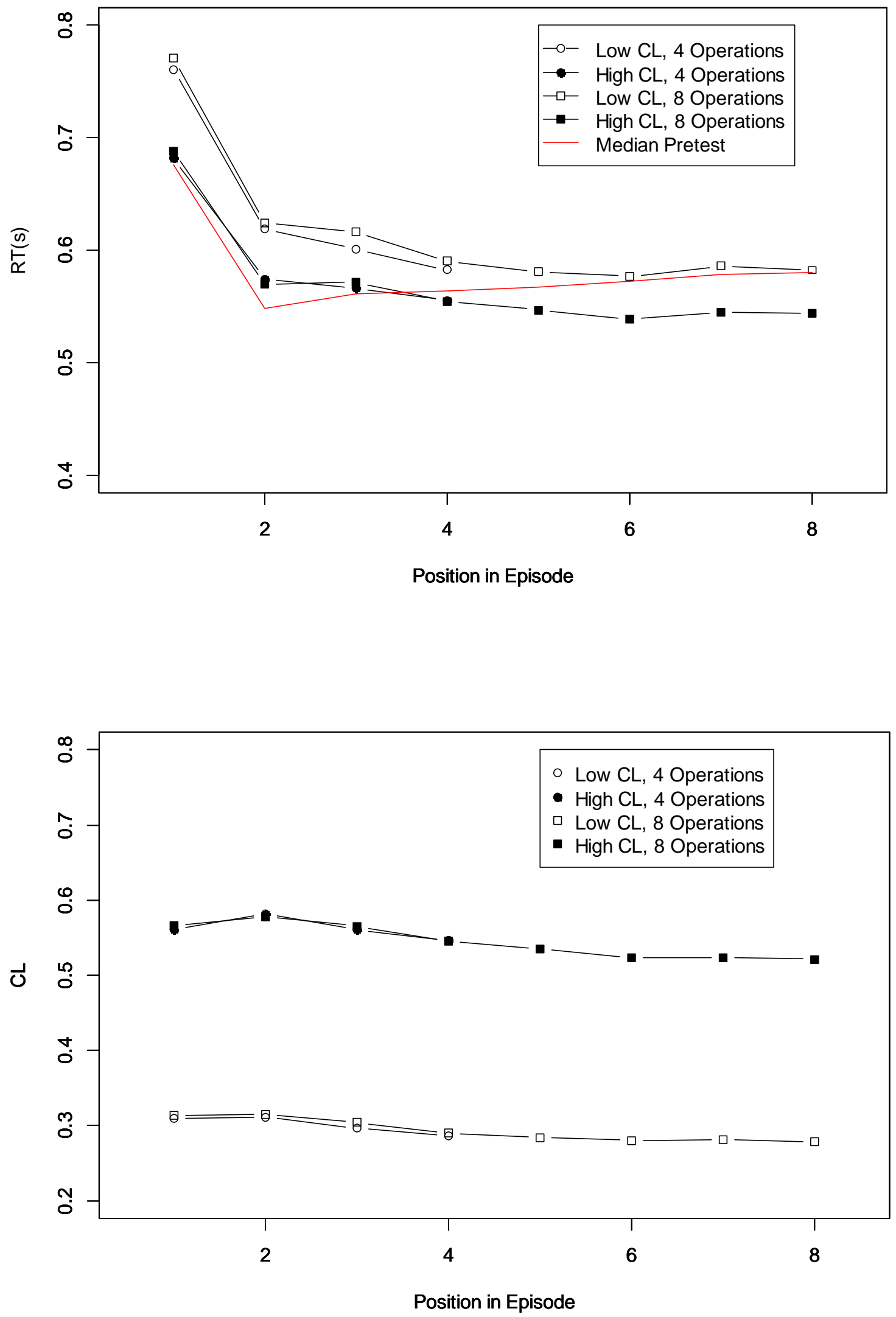
Figure 5

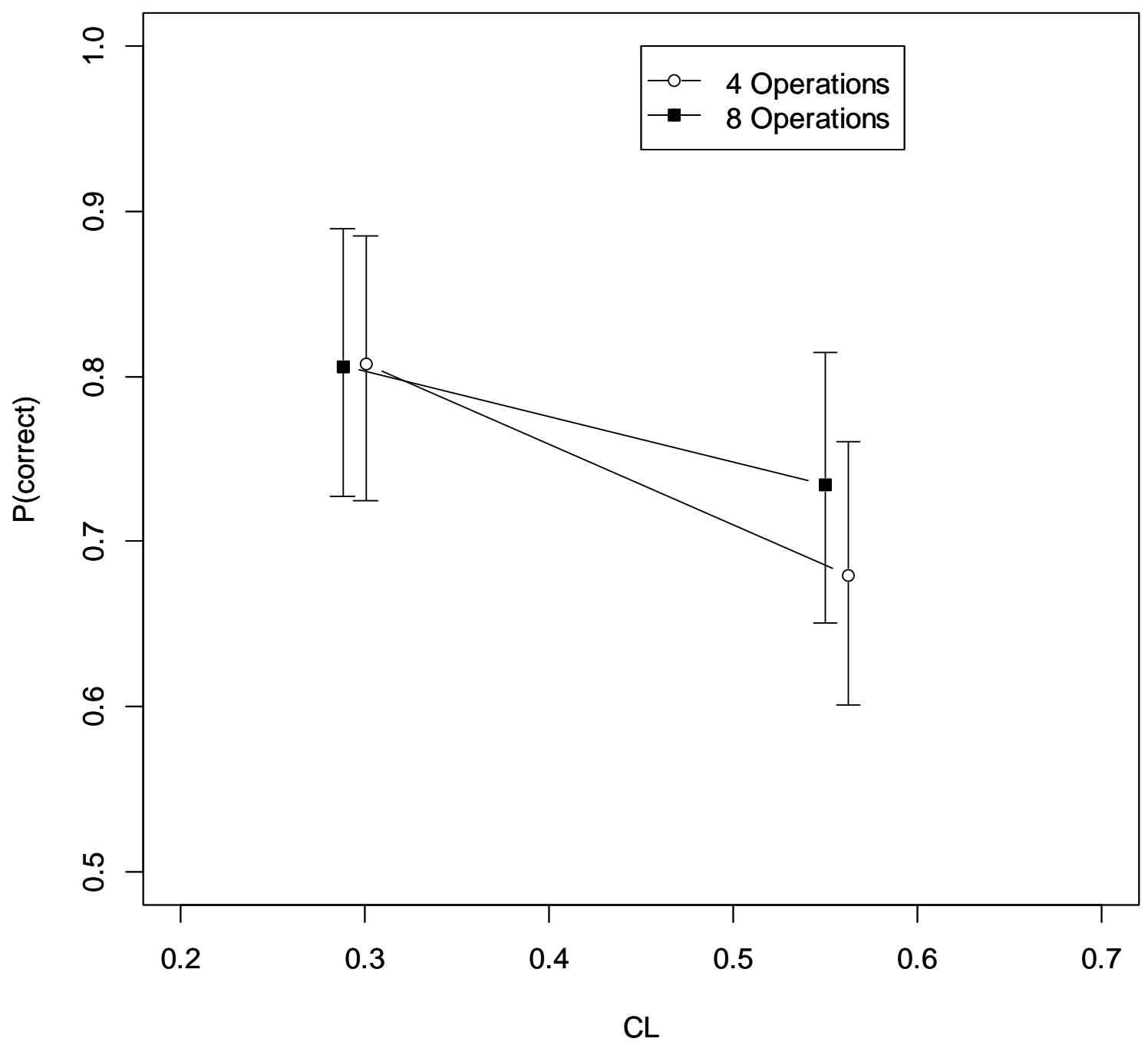


Figure 6

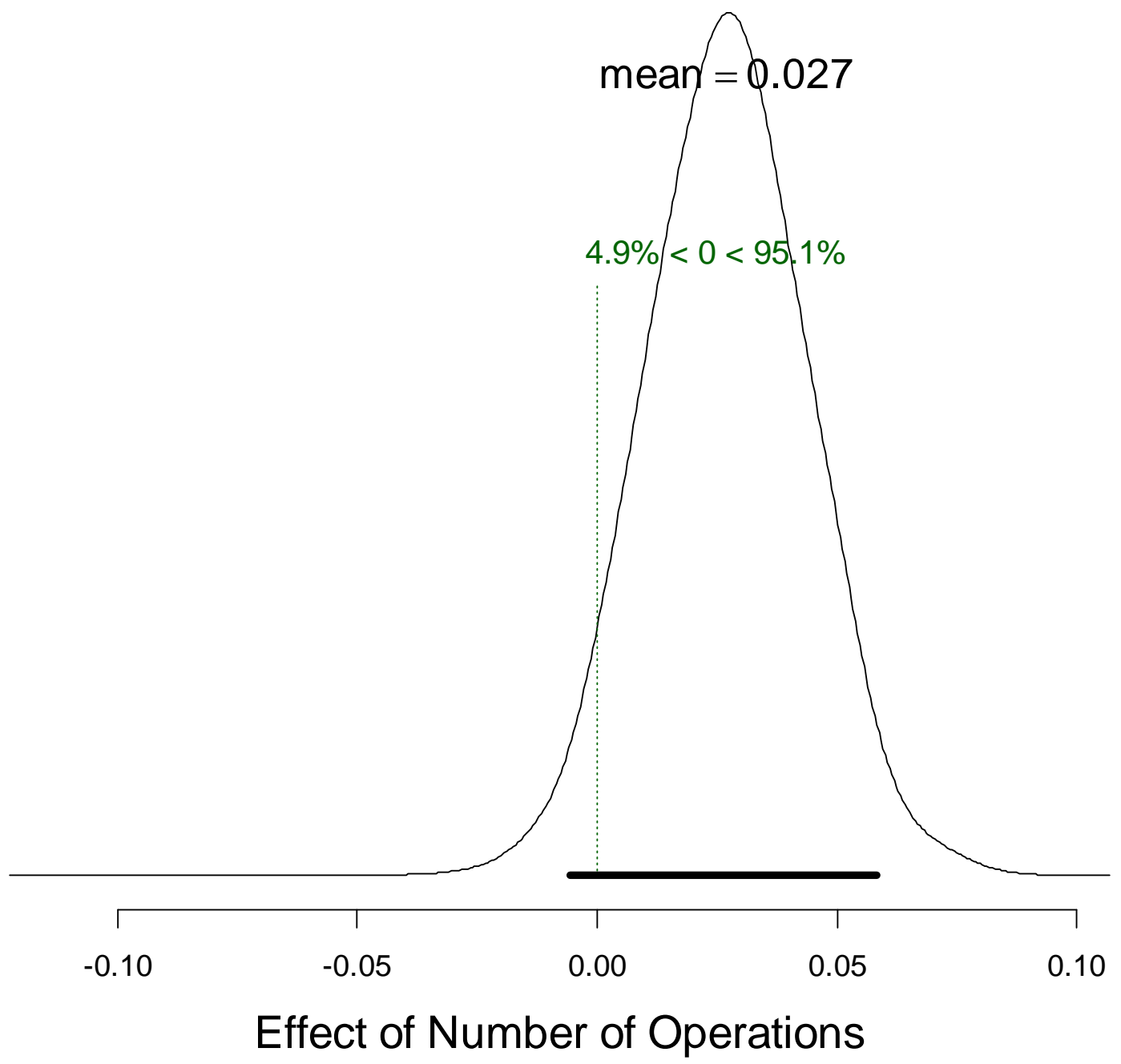


Figure 7
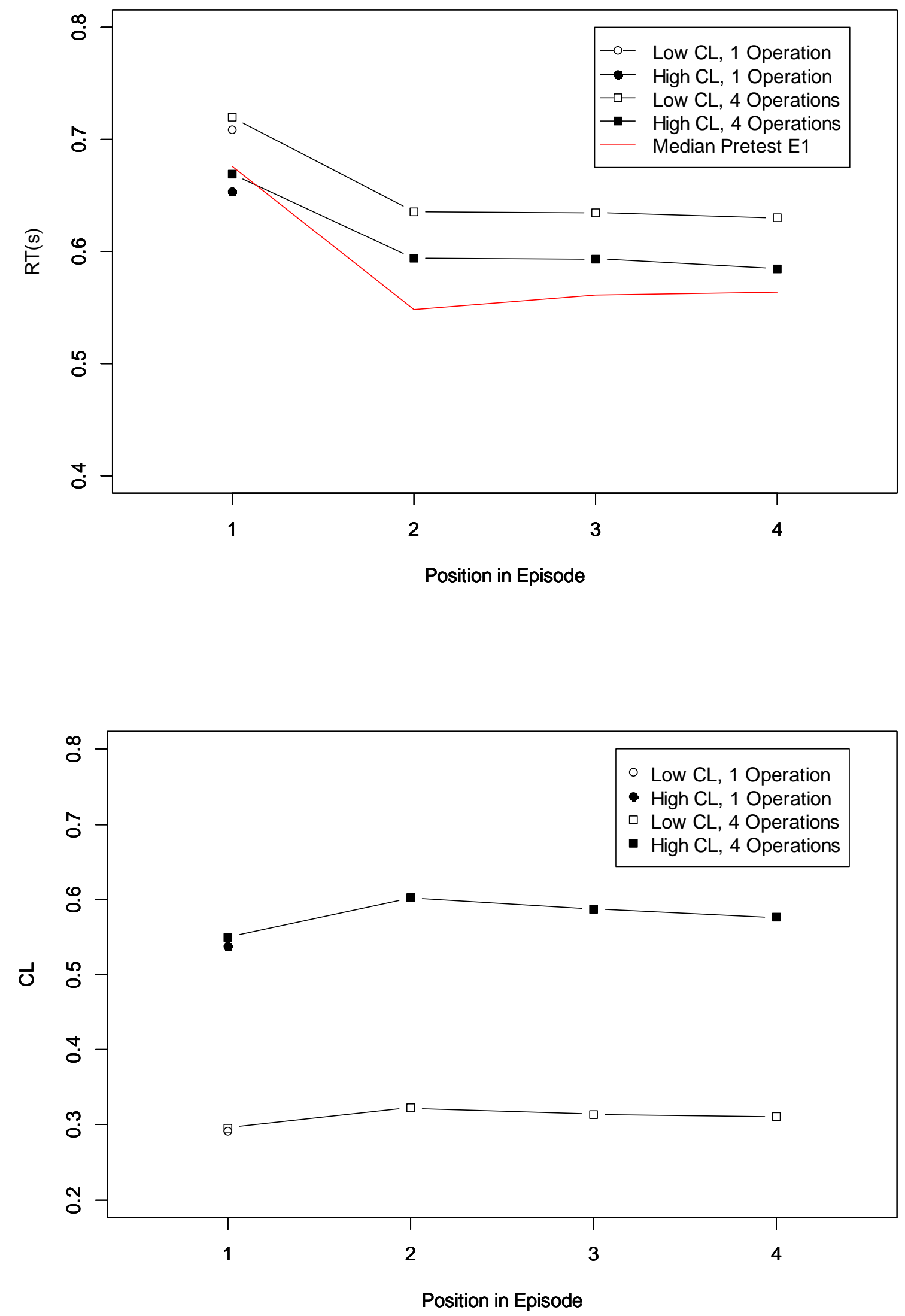
Figure 8

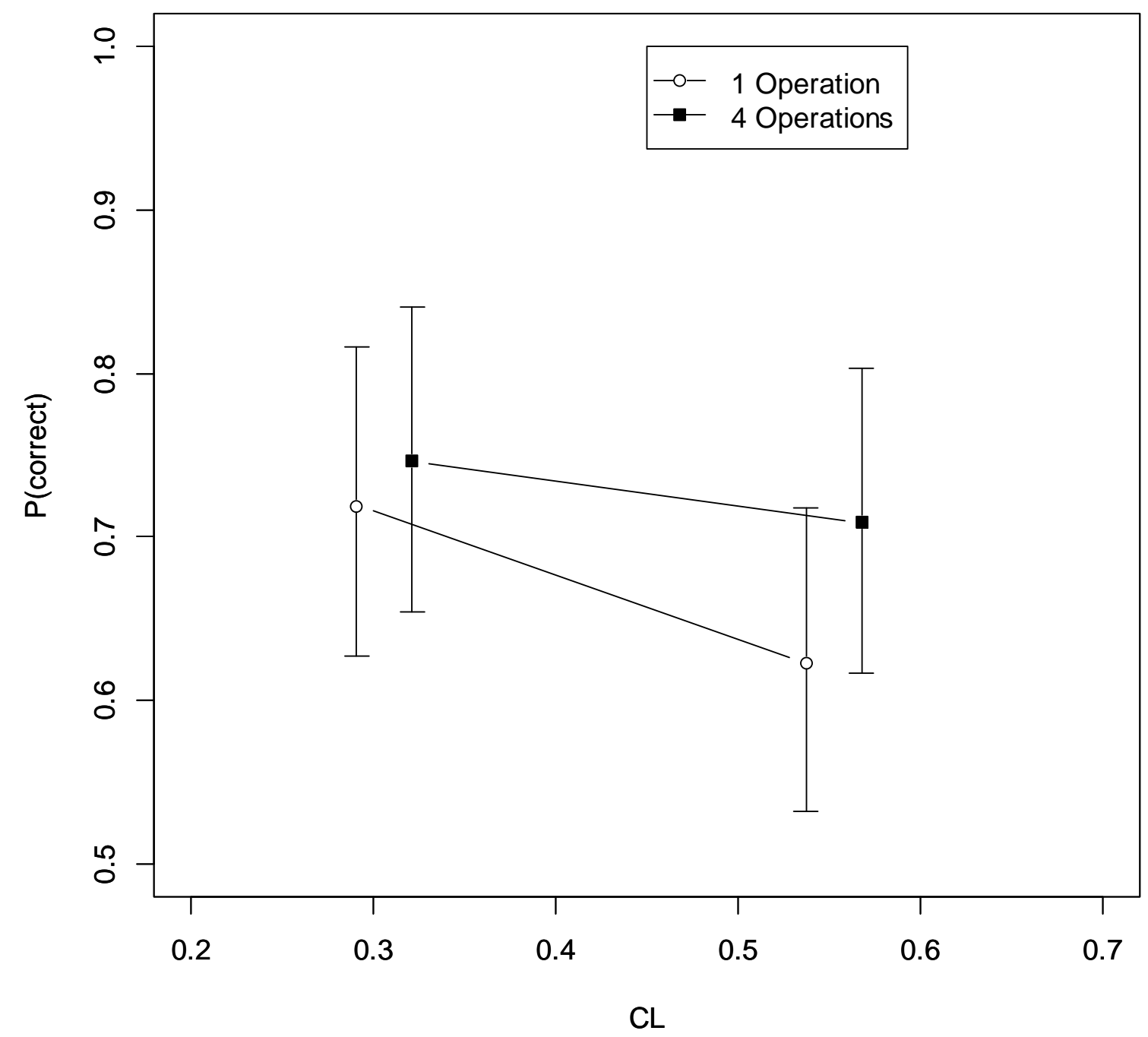


Figure 9

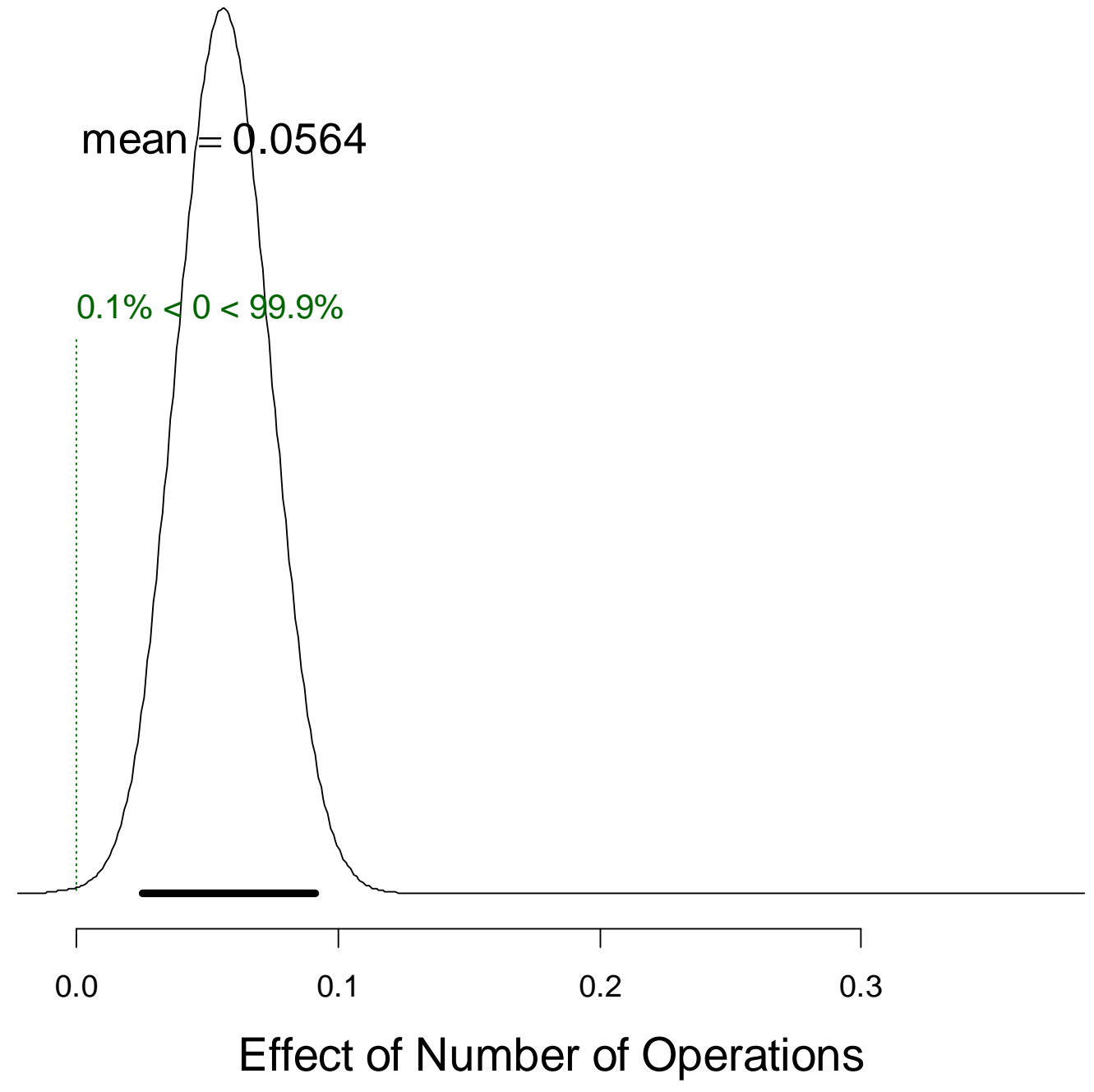


Figure 10

Predictions from TBRS*

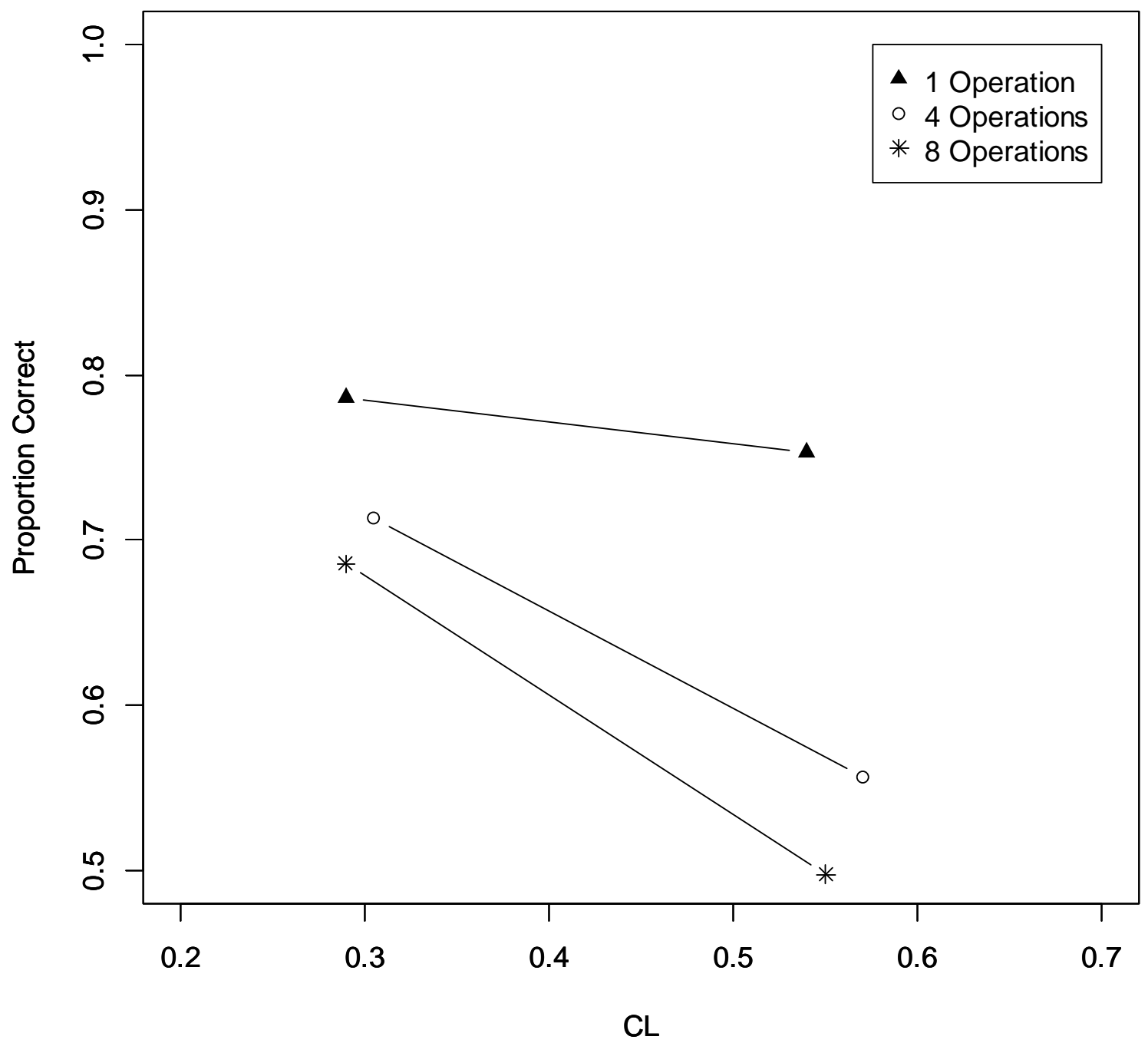


Figure 11
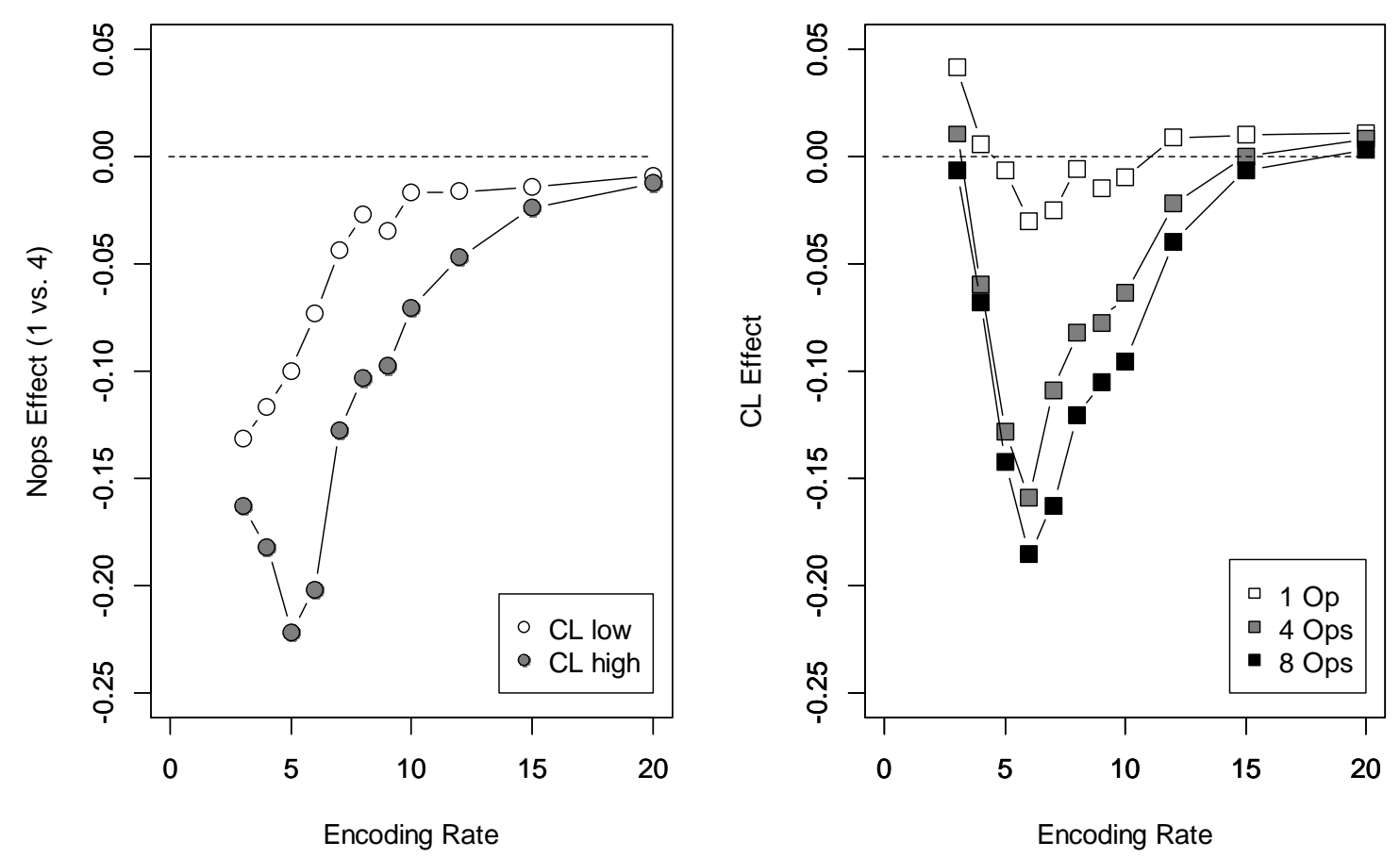
Figure 12
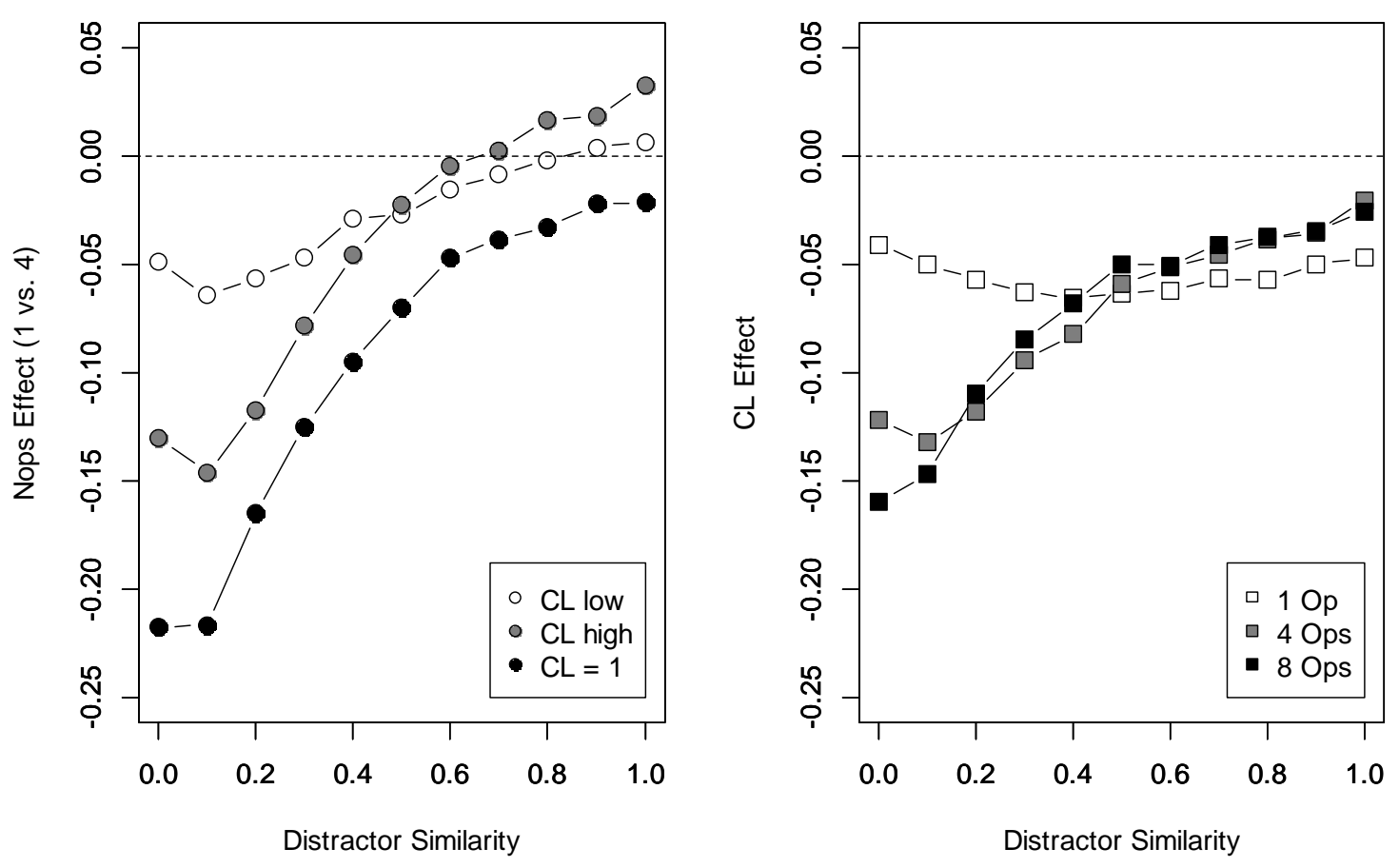
Figure 13

Predictions from SOB-CS

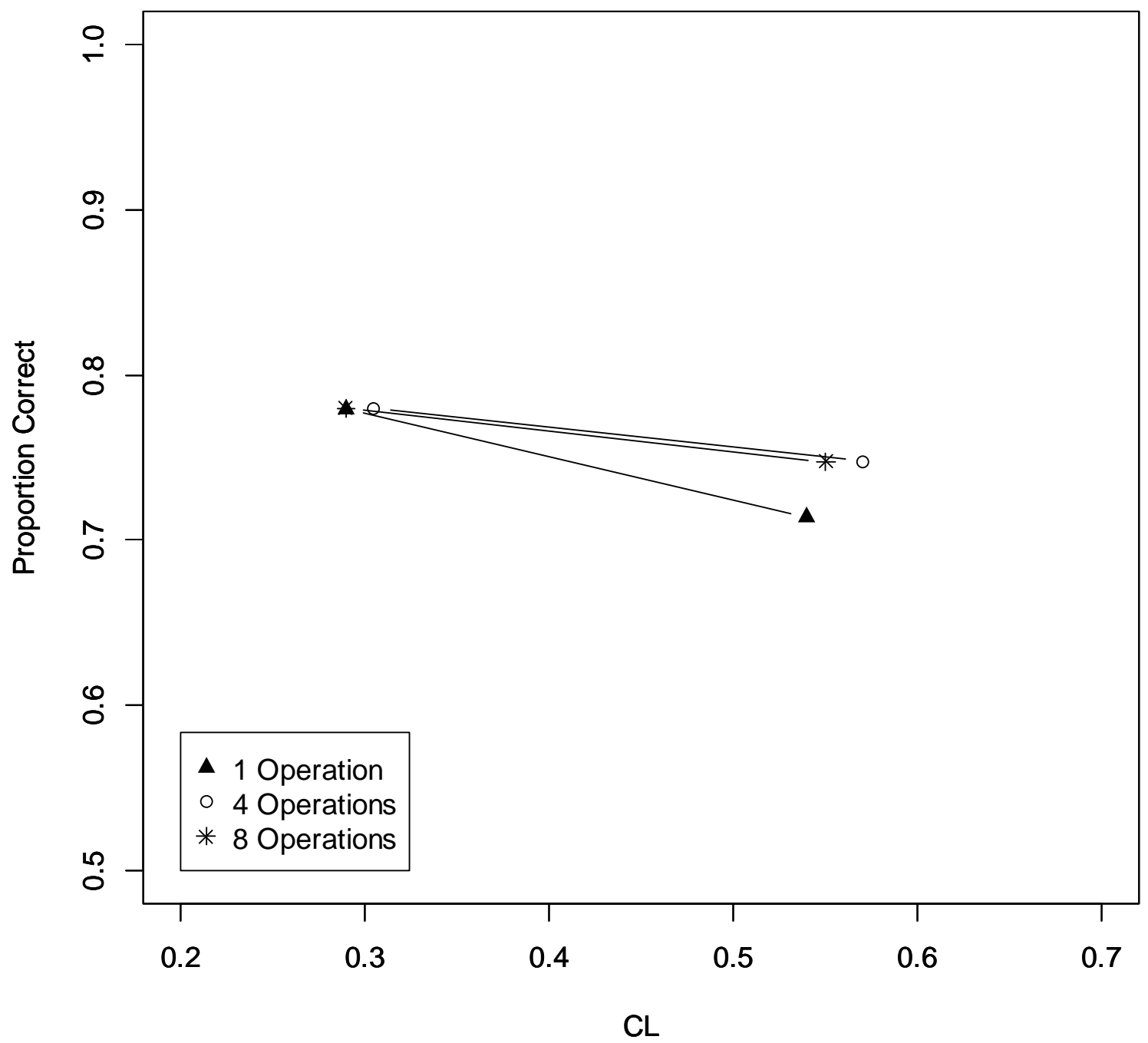


Figure A1

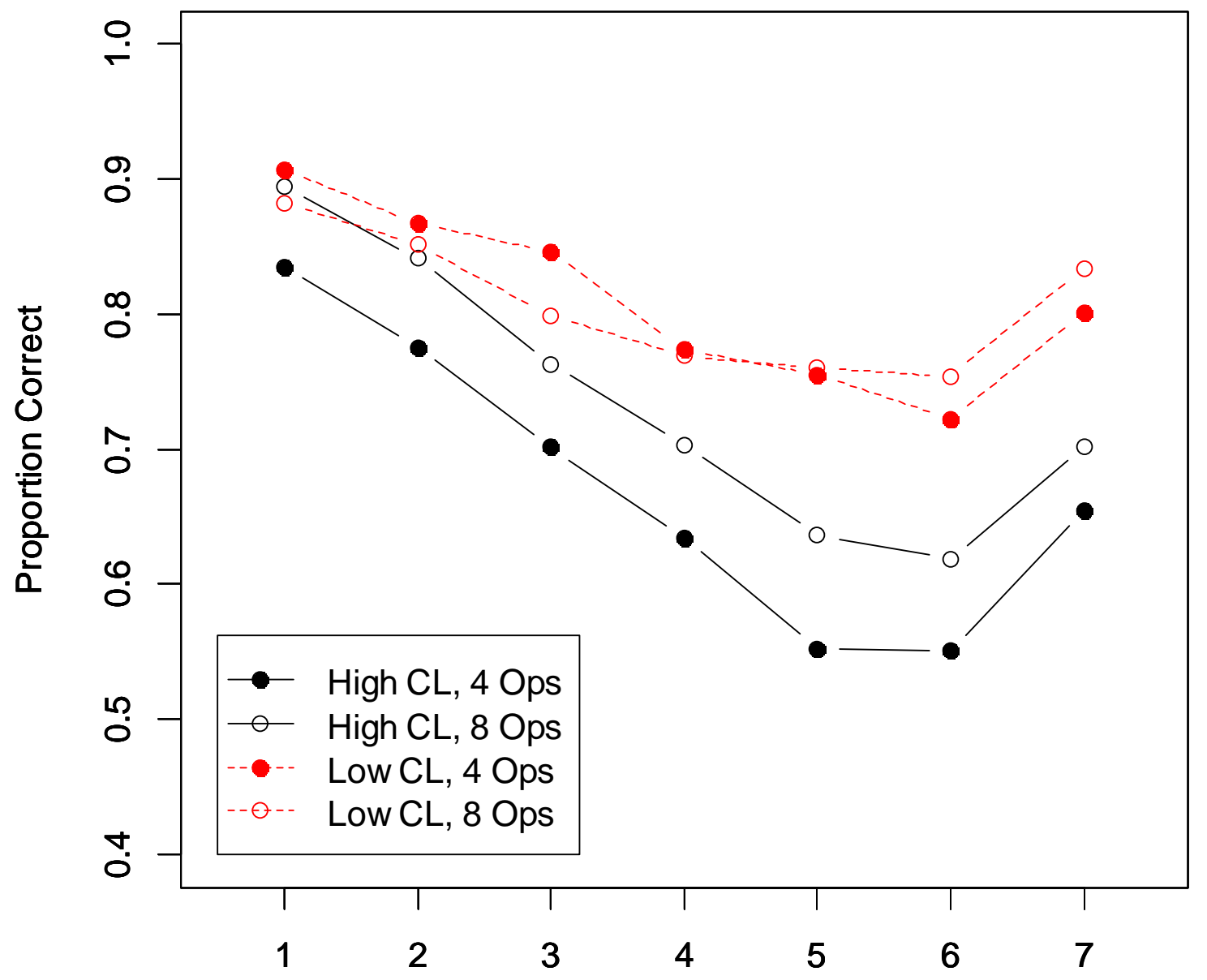

Serial Position 
Figure A2

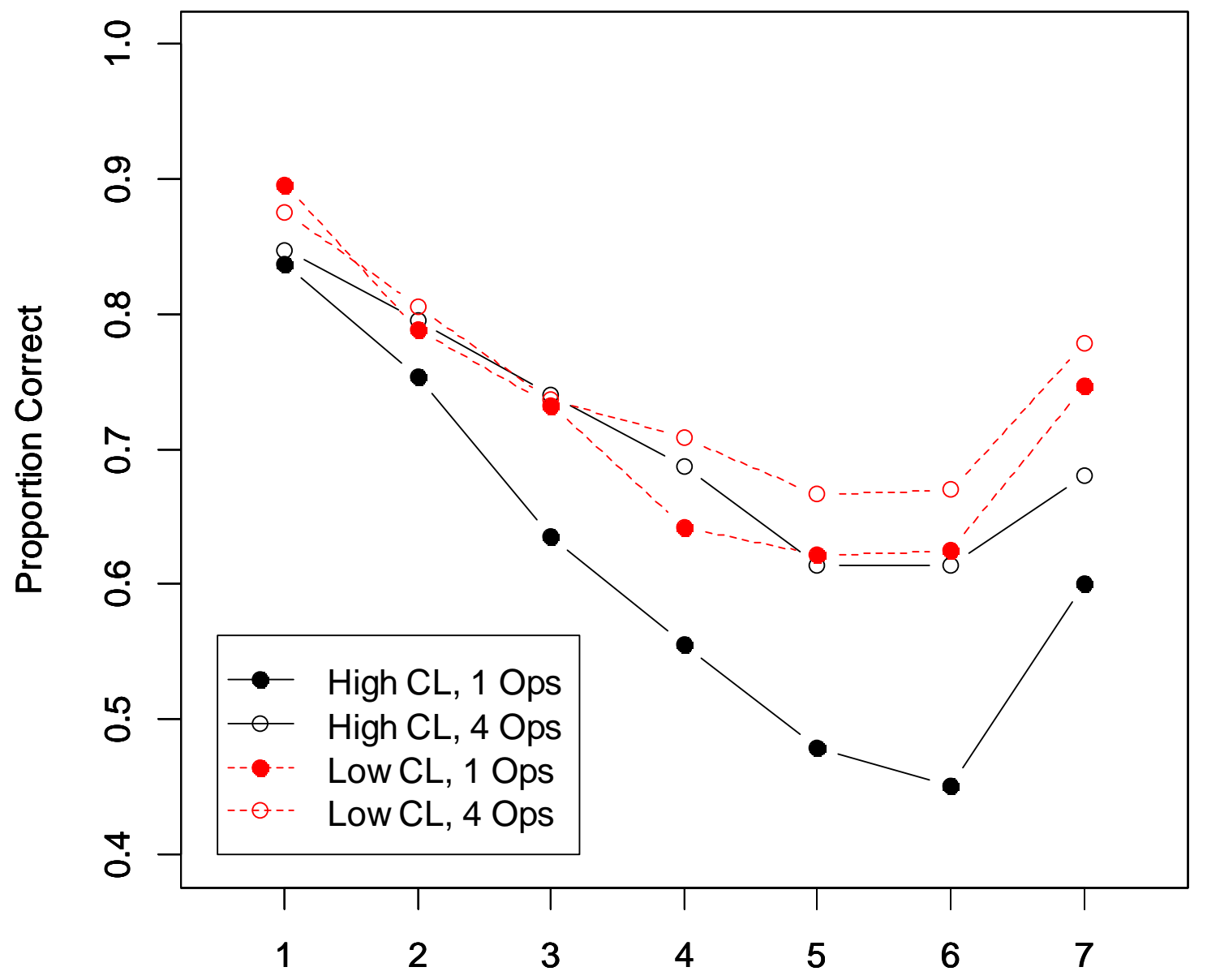

Serial Position 